\title{
Heating (Gapless) Color-Flavor Locked Quark Matter
}

\author{
Kenji Fukushima, ${ }^{1,2}$ Chris Kouvaris, ${ }^{1}$ and Krishna Rajagopal ${ }^{1}$ \\ ${ }^{1}$ Center for Theoretical Physics, Massachusetts Institute of Technology, Cambridge, MA 02139, USA \\ ${ }^{2}$ Department of Physics, University of Tokyo, 7-3-1 Hongo, Tokyo 113-0033, Japan
}

(Dated: August 25, 2004)

\begin{abstract}
We explore the phase diagram of neutral quark matter at high baryon density as a function of the temperature $T$ and the strange quark mass $M_{s}$. At $T=0$, there is a sharp distinction between the insulating color-flavor locked (CFL) phase, which occurs where $M_{s}^{2} / \mu<2 \Delta$, and the metallic gapless CFL phase, which occurs at larger $M_{s}^{2} / \mu$. Here, $\mu$ is the chemical potential for quark number and $\Delta$ is the gap in the CFL phase. We find this distinction blurred at $T \neq 0$, as the CFL phase undergoes an insulator-to-metal crossover when it is heated. We present an analytic treatment of this crossover. At higher temperatures, we map out the phase transition lines at which the gap parameters $\Delta_{1}, \Delta_{2}$ and $\Delta_{3}$ describing $d s$-pairing, $u s$-pairing and $u d$-pairing respectively, go to zero in an NJL model. For small values of $M_{s}^{2} / \mu$, we find that $\Delta_{2}$ vanishes first, then $\Delta_{1}$, then $\Delta_{3}$. We find agreement with a previous Ginzburg-Landau analysis of the form of these transitions and find quantitative agreement with results obtained in full QCD at asymptotic density for ratios of coefficients in the Ginzburg-Landau potential. At larger $M_{s}^{2} / \mu$, we find that $\Delta_{1}$ vanishes first, then $\Delta_{2}$, then $\Delta_{3}$. Hence, we find a "doubly critical" point in the $\left(M_{s}^{2} / \mu, T\right)$-plane at which two lines of second order phase transitions $\left(\Delta_{1} \rightarrow 0\right.$ and $\left.\Delta_{2} \rightarrow 0\right)$ cross. Because we do not make any small- $M_{s}$ approximation, if we choose a relatively strong coupling leading to large gap parameters, we are able to pursue the analysis of the phase diagram all the way up to such large values of $M_{s}$ that there are no strange quarks present.
\end{abstract}

PACS numbers: 12.38.-t, 25.75.Nq

\section{INTRODUCTION}

Our understanding of the properties of matter at high baryon number density has developed rapidly in the last several years. At any densities that are high enough that nucleons are crushed into quark matter, the quark matter that results must, at sufficiently low temperatures, be in one of a family of color superconducting phases [1]. The essence of color superconductivity is quark pairing, driven by the BCS mechanism which operates whenever there is an attractive interaction between fermions at a Fermi surface. The QCD quark-quark interaction is strong and is attractive between quarks that are antisymmetric in color, so we expect cold dense quark matter to generically exhibit color superconductivity. Color superconducting quark matter may well occur in the cores of compact stars.

It is by now well-established that at asymptotic densities, where the up, down and strange quarks can be treated on an equal footing and the disruptive effects of the strange quark mass can be neglected, quark matter is in the color-flavor locked (CFL) phase, in which quarks of all three colors and all three flavors form Cooper pairs [2]. The CFL phase is a color superconductor but is an electromagnetic insulator, with zero electron density.

To describe quark matter as may exist in the cores of compact stars, we need consider quark chemical potentials $\mu$ of order $500 \mathrm{MeV}$ at most, meaning that the strange quark mass $M_{s}$ must be included: it is expected to be density dependent, lying between the current mass $\sim 100 \mathrm{MeV}$ and the vacuum constituent quark mass $\sim$ $500 \mathrm{MeV}$. In bulk matter, as is relevant for compact stars where we are interested in kilometer-scale volumes, we must furthermore require electromagnetic and color neutrality [3, 4] (possibly via mixing of oppositely charged phases) and allow for equilibration under the weak interactions. All these factors work to pull apart the Fermi momenta of the cross-species pairing that characterizes color-flavor locking. At the highest densities, we expect CFL pairing, but as the density decreases the combination of nonzero $M_{s}$ and the constraints of neutrality put greater and greater stress on cross-species pairing, and reduce the excitation energies of those fermionic quasiparticles whose excitation would serve to ease the stress by breaking pairs [5].

If we imagine beginning in the CFL phase at asymptotic density, reducing the density, and assume that CFL pairing is disrupted by the heaviness of the strange quark before color superconducting quark matter is superseded by the hadronic phase, the next phase down in density is the gapless CFL phase [5, 6. In this phase, quarks of all three colors and all three flavors still form Cooper pairs, but there are regions of momentum space in which certain quarks do not succeed in pairing, and these regions are bounded by momenta at which certain fermionic quasiparticles are gapless. The material is an electromagnetic conductor, with a nonzero electron density.

Our goal in this paper is to map the phase diagram of neutral dense quark matter at nonzero temperature, answering the question of what phases and phase transitions result when CFL or gCFL quark matter is heated. In the case of CFL quark matter with strange quark mass $M_{s}=0$, this question has been answered in Ref. [7]. In this most symmetric setting, there is a single phase transition at a temperature $T_{c}^{\mathrm{CFL}}$ below which there is CFL 
pairing and above which there is no pairing. In mean field theory,

$$
T_{c}^{\mathrm{CFL}}=2^{\frac{1}{3}} \frac{e^{\gamma}}{\pi} \Delta_{0},
$$

where $\Delta_{0}$ is the CFL gap parameter at $T=0$, estimated to be of order 10 to $100 \mathrm{MeV}$ [1]. The enhancement of $T_{c} / \Delta_{0}$ by a factor of $2^{1 / 3}$ over the standard BCS value (which our results confirm) originates in the fact that in the CFL phase with $M_{s}=0$ there are eight fermionic quasiparticles with gap $\Delta_{0}$ and one with gap $2 \Delta_{0}$ [7]. As at any phase transition at which a superconducting order parameter melts, gauge field fluctuations that are neglected in mean field theory can elevate $T_{c}$ and render the transition first order [8]. Because the gauge coupling in QCD is strong, these effects are significant 9, 10]. They have been evaluated to date only at $M_{s}=0[9,10]$. Once $M_{s} \neq 0$, the mean field analysis alone becomes rather involved and we shall therefore leave the inclusion of fluctuations to future work.

To see why $M_{s} \neq 0$ results in an intricate phase diagram at nonzero temperature, we must introduce the pairing ansatz that we shall use throughout this paper [2, 5, [6, 11]:

$$
\left\langle\psi_{a}^{\alpha} C \gamma_{5} \psi_{b}^{\beta}\right\rangle \sim \Delta_{1} \epsilon^{\alpha \beta 1} \epsilon_{a b 1}+\Delta_{2} \epsilon^{\alpha \beta 2} \epsilon_{a b 2}+\Delta_{3} \epsilon^{\alpha \beta 3} \epsilon_{a b 3}
$$

Here $\psi_{a}^{\alpha}$ is a quark of color $\alpha=(r, g, b)$ and flavor $a=(u, d, s)$; the condensate is a Lorentz scalar, antisymmetric in Dirac indices, antisymmetric in color (the channel with the strongest attraction between quarks), and consequently antisymmetric in flavor. The gap parameters $\Delta_{1}, \Delta_{2}$ and $\Delta_{3}$ describe down-strange, up-strange and up-down Cooper pairs, respectively. At $T=0$, there is an insulator-metal transition at $M_{s}^{2} / \mu \simeq 2 \Delta_{1}$, at which the CFL insulator with $\Delta_{3} \simeq \Delta_{2}=\Delta_{1}$ is replaced by the gapless CFL metal, which has $\Delta_{3}>\Delta_{2}>$ $\Delta_{1}>0$ [5, 6]. (If the CFL phase is augmented by a $K^{0}$ condensate [12], the CFL $\rightarrow$ gCFL transition is delayed to $M_{s}^{2} / \mu \simeq 8 \Delta_{1} / 3$ [13].) An analogous zero temperature metal insulator transition has been analyzed in Ref. 14].

At $M_{s}=0, \Delta_{1}=\Delta_{2}=\Delta_{3}=\Delta_{\mathrm{CFL}}$, and $\Delta_{\mathrm{CFL}}$ decreases from $\Delta_{0}$ at $T=0$ to 0 at $T_{c}^{\mathrm{CFL}}$. As soon as $M_{s} \neq 0$, however, we can expect that $\Delta_{1}, \Delta_{2}$ and $\Delta_{3}$ do not all vanish at the same temperature. This expectation is evident for the gCFL phase, which has $\Delta_{3}>\Delta_{2}>\Delta_{1}>0$ already at $T=0$. We shall see that it also applies to the CFL phase with $M_{s} \neq 0$.

The distinction between an insulator and a metal is sharp only at $T=0$. At any nonzero temperature, there will be some nonzero density of thermally excited fermionic quasiparticles, some of which are charged. This means that the CFL $\rightarrow$ gCFL "transition" should be a crossover at any nonzero temperature [15]. The CFL phase at $T=0$ with $M_{s} \neq 0$ has fermionic quasiparticles with opposite charges whose excitation energies differ. This means that upon heating this phase, the chemical potentials needed to maintain neutrality are not the same as at zero temperature. Adjusting the chemical potentials feeds back into the gap equations for $\Delta_{1}, \Delta_{2}$ and $\Delta_{3}$ differently, and these gap parameters can therefore have different $T$-dependence. If we start in the CFL phase at $T=0$, heat, then increase $M_{s}^{2} / \mu$ above that for the zero temperature $\mathrm{CFL} \rightarrow \mathrm{gCFL}$ transition, and then cool back to $T=0$ we can go from CFL to gCFL without ever crossing a phase boundary. We illustrate this by showing an example phase diagram in Fig. 1.

The purpose of this paper is to derive and understand the phase diagram of Fig. 1 We shall present the model and approximations that we use in Section III We follow the conventions for naming phases used in previous literature:

$$
\begin{array}{ll}
\Delta_{1}, \Delta_{2}, \Delta_{3} \neq 0 & \text { (g)CFL } \\
\Delta_{1}=0, \quad \Delta_{2}, \Delta_{3} \neq 0 & \text { uSC } \\
\Delta_{2}=0, \quad \Delta_{1}, \Delta_{3} \neq 0 & \text { dSC } \\
\Delta_{1}=\Delta_{2}=0, \quad \Delta_{3} \neq 0 & \text { (g) } 2 \text { SC } \\
\Delta_{1}=\Delta_{2}=\Delta_{3}=0 & \text { UQM },
\end{array}
$$

with "UQM" meaning unpaired quark matter. The origin of the remaining names is that in the $2 \mathrm{SC}$ phase, only quarks of two flavors and two colors pair, whereas in the uSC (or dSC) phase, all $u$ (or all $d$ ) quarks pair 16]. The phase diagram features three lines denoting second order phase transitions at which $\Delta_{1}, \Delta_{2}$ or $\Delta_{3}$ vanish. At $M_{s}=0$, all three gaps vanish at the same temperature but at generic values of $M_{s}$, there are three distinct transition temperatures. Two of these lines cross at a point denoted by the circle: at this "doubly critical point", $\Delta_{1}$ and $\Delta_{2}$ vanish at the same temperature. The phase diagram is intricate, and one natural question is to what extent its features are generic. We address this by varying parameters, as we shall discuss in Sections III and IV We shall find that the physics at large $M_{s}^{2} / \mu$, where we find a first order phase transition in Fig. [1 is not generic, changing qualitatively for larger values of $\Delta_{0}$. We find that the shape of the second order phase boundaries separating the CFL, dSC, uSC and 2SC phases is generic, as is the existence of the doubly critical point.

Physics in the vicinity of any of these phase transitions can be analyzed using a Ginzburg-Landau approximation, in which the relevant $\Delta$ or $\Delta$ 's are taken to be small. This analysis has been performed at $M_{s}=0$ in Refs. 3, 9, 17] and at small but nonzero $M_{s}$ in Ref. [16]. We shall show in Section DI that our numerical results for the three phase transition lines at small $M_{s}^{2} / \mu \Delta_{0}$ are in quantitative agreement with the Ginzburg-Landau approximation, and shall show that the ratios of coefficients in the Ginzburg-Landau potential that we obtain in our model agree quite well with those obtained in full QCD at higher densities. At small $M_{s}^{2} / \mu \Delta_{0}$, the region of the phase diagram where the Ginzburg-Landau analysis applies near $T_{c}, \Delta_{2}$ vanishes at a lower critical temperature than $\Delta_{1}$. In heating gCFL quark matter, however, we expect $\Delta_{1}$ to vanish first because it is already much smaller at $T=0$. Our model analysis can be extended to larger 


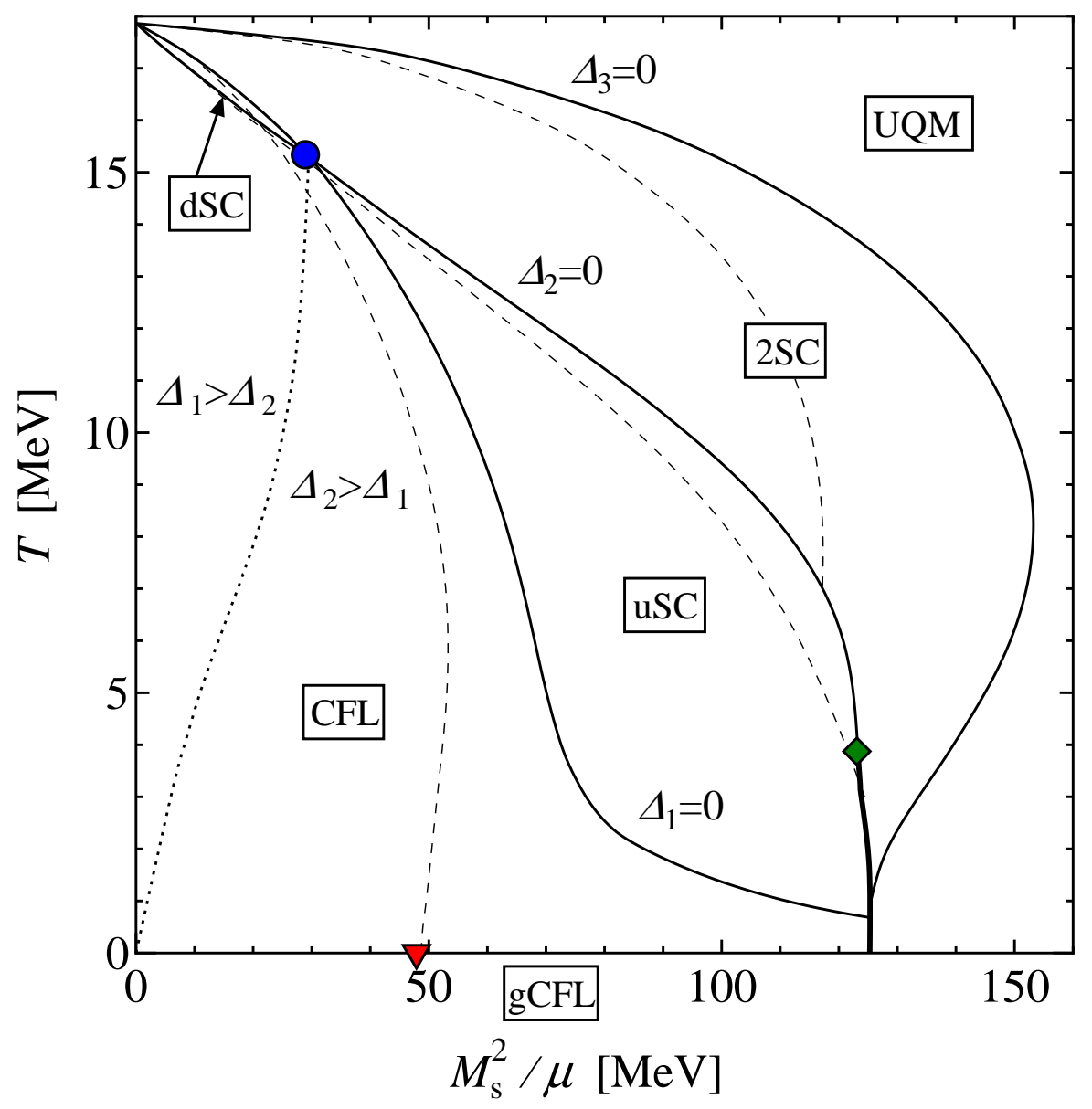

FIG. 1: Phase diagram of dense neutral quark matter in the $\left(M_{s}^{2} / \mu, T\right)$ plane. Only the solid curves represent phase transitions. The thin solid curves denote mean field second order phase transitions at which $\Delta_{1}$ or $\Delta_{2}$ or $\Delta_{3}$ vanish. Two of these lines cross at the circle, which we call a "doubly critical" point. The heavy solid line is a first order phase transition that ends at a tricritical point denoted by the diamond. The dashed curves indicate locations where, as $M_{s}^{2} / \mu$ is increased, new gapless modes appear. At any nonzero temperature, however, there is no physical distinction between a phase with truly gapless modes and one in which the same modes have excitation energies of order $T$. The dashed curves therefore have physical significance only where they cross $T=0$. In this phase diagram, this occurs only at the triangle, which denotes the zero temperature $\mathrm{CFL} \rightarrow \mathrm{gCFL}$ transition, which is a quantum critical point. The dotted line separates the $\Delta_{1}>\Delta_{2}$ and $\Delta_{2}>\Delta_{1}$ regions. This phase diagram is drawn for $\mu=500 \mathrm{MeV}$, with $M_{s}$ and $T$ varying, and with the quark-quark interaction strength fixed and chosen such that the CFL gap is $\Delta_{0}=25 \mathrm{MeV}$ at $T=0$. We shall describe the model and approximations within which this phase diagram has been obtained in Section II and describe the results summarized by this diagram in Sections III and IV

$M_{s}$ and smaller $T$ than the Ginzburg-Landau analysis, allowing us to see how the phase diagram fits together, consistent with this expectation.

A phase diagram similar to ours was obtained previously in Ref. [15], but our results differ in a crucial, qualitative respect at low $M_{s}^{2} / \mu$, where we believe that our results are robust: the authors of Ref. 15] found that $\Delta_{1}$ always vanishes at a lower temperature than $\Delta_{2}$, meaning that there is no dSC region on their phase diagram. This also disagrees with the Ginzburg-Landau analysis [47]. We shall detail our approximations in Section [II] but we note already here that whereas in Refs. [5, 6, 15] $M_{s}$ was assumed to be much smaller than $\mu$, we do not make such an approximation. This likely explains the differ- ences between our results and those of Ref. 15] at large $M_{s}^{2} / \mu$.

Although not associated with a phase transition, it is interesting to ask how the CFL phase ceases to be an insulator as it is heated. This must happen, given the phase diagram that we and the authors of Ref. 15] find, but how? At small $T$, the number density of charged quark quasiparticles in the CFL phase is exponentially small. Because of the interplay between $M_{s} \neq 0$ and the constraints of neutrality, the excitation energies of oppositely charged quasiparticles are not the same and these thermally excited quasiparticles have a net charge which must be balanced by a nonzero electron density of order $\mu_{e}^{3}$, where $\mu_{e}$ is the electron chemical potential. Because 
the quasiparticle densities are proportional to the quark Fermi surface area $\sim \mu^{2}$, where $\mu \gg \mu_{e}$, we show that $\mu_{e}$ ceases to be exponentially suppressed - rising rapidly to $\mu_{e} \sim M_{s}^{2} / 4 \mu$-in an insulator-metal crossover that occurs in a narrow, and quite low, range of temperatures. We describe this crossover analytically in Section $\nabla$ There are other charged excitations in the CFL phase, namely the pseudo-Nambu-Goldstone bosons, that are excited when the system is heated. We argue quantitatively that their effects are small.

In Section $\amalg$ we detail our model and approximations, stressing also those approximations used in previous work that we have not made. In Section [III we review results at $T=0$. In Section IV] we present our results at $T \neq 0$, analyzing the phase diagram in Fig. 1 and that for other values of $\Delta_{0}$. In Section $\nabla$ we analyze the insulator-metal crossover that occurs when the CFL phase is heated. In Section VI] we make the connection between our work and the Ginzburg-Landau analysis quantitative. We conclude in Section VII

\section{MODEL AND APPROXIMATIONS}

\section{A. Pairing ansatz for gap parameters}

We assume that the predominant diquark condensate is a Lorentz scalar (antisymmetric in Dirac indices), is antisymmetric in color (as is favored energetically), and consequently is antisymmetric in flavor. That is, we assume a condensate of the form (2). The gap parameters $\Delta_{1}, \Delta_{2}$ and $\Delta_{3}$ describe a $9 \times 9$ matrix in color-flavor space that, in the basis $(r u, g d, b s, r d, g u, r s, b u, g s, b d)$, takes the form

$\boldsymbol{\Delta}=\gamma_{5} \cdot\left(\begin{array}{ccccccccc}0 & \Delta_{3} & \Delta_{2} & 0 & 0 & 0 & 0 & 0 & 0 \\ \Delta_{3} & 0 & \Delta_{1} & 0 & 0 & 0 & 0 & 0 & 0 \\ \Delta_{2} & \Delta_{1} & 0 & 0 & 0 & 0 & 0 & 0 & 0 \\ 0 & 0 & 0 & 0 & -\Delta_{3} & 0 & 0 & 0 & 0 \\ 0 & 0 & 0 & -\Delta_{3} & 0 & 0 & 0 & 0 & 0 \\ 0 & 0 & 0 & 0 & 0 & 0 & -\Delta_{2} & 0 & 0 \\ 0 & 0 & 0 & 0 & 0 & -\Delta_{2} & 0 & 0 & 0 \\ 0 & 0 & 0 & 0 & 0 & 0 & 0 & 0 & -\Delta_{1} \\ 0 & 0 & 0 & 0 & 0 & 0 & 0 & -\Delta_{1} & 0\end{array}\right)$

We see that $(g s, b d),(r s, b u)$ and $(r d, g u)$ quarks pair with gap parameters $\Delta_{1}, \Delta_{2}$ and $\Delta_{3}$ respectively, while the $(r u, g d, b s)$ quarks pair among each other involving all the $\Delta$ 's. The most important physics that we are leaving out by making this ansatz is pairing in which the Cooper pairs are symmetric in color, and therefore also in flavor. Diquark condensates of this form break no new symmetries, and therefore must arise in the CFL phase [2, 18]. However because the QCD interaction is repulsive between quarks that are symmetric in color these condensates are numerically insignificant [2, 15, 18].

\section{B. CFL symmetries and excitations}

To set the stage for our analysis, and to introduce the excitations that shall concern us, we briefly summarize the properties of the CFL phase 2]. If we set all three quark masses to zero, the diquark condensate in the CFL phase (which then has $\Delta_{1}=\Delta_{2}=\Delta_{3}$ ) spontaneously breaks the full symmetry group of QCD,

$$
\begin{aligned}
{\left[S U(3)_{\text {color }}\right] } & \times \underbrace{S U(3)_{L} \times S U(3)_{R}}_{\supset\left[U(1)_{Q}\right]} \times U(1)_{B} \\
& \rightarrow \underbrace{S U(3)_{C+L+R}}_{\supset\left[U(1)_{\tilde{Q}}\right]} \times \mathbb{Z}_{2}
\end{aligned}
$$

where $S U(3)_{\text {color }}$ and electromagnetism $U(1)_{Q}$ are gauged, and the unbroken $S U(3)_{c+L+R}$ subgroup consists of flavor rotations of the left and right quarks with equal and opposite color rotations, and contains an unbroken gauged "rotated electromagnetism" $U(1)_{\tilde{Q}}[2,[19]$. The CFL phase has the largest possible unbroken symmetry consistent with diquark condensation, achieved by having all nine quarks participate equally in the pairing, and this gives the maximal pairing free energy benefit. Not surprisingly, $a b$ initio calculations valid at asymptotic densities confirm that the CFL phase is the ground state of QCD in the high density limit [1, 20].

The axial flavor $U(1)_{A}$ rotations are not a symmetry of QCD, as they are explicitly broken at all densities by instanton effects. At asymptotic densities, these effects become small but at accessible densities they cannot be neglected 2, 21, 22, 23, 24], and they shall play an important role in Section $\mathrm{VA}$

In the limit of three massless quarks described above there are 17 broken symmetry generators in the CFL phase, 8 of which become longitudinal components of massive gauge bosons and 9 of which remain as NambuGoldstone bosons. The massive gauge bosons have excitation energies of order $g \mu$, where the QCD gauge coupling $g$ is not small, and we neglect them throughout. In the real world, with its two light quark flavors with masses $\lesssim 10 \mathrm{MeV}$, and a medium-weight flavor, the strange quark, with mass $\gtrsim 100 \mathrm{MeV}$, the eight Nambu-Goldstone bosons coming from the breaking of chiral symmetries acquire masses 1, 2, 23, 24, [25, [26], and furthermore the CFL condensate may rotate in the $K^{0}$-direction within the manifold describing these mesons [12]. We neglect the possibility of $K^{0}$ condensation throughout. We shall see in Section $\nabla A$ that this corresponds to assuming that the instanton contribution to the $K^{0}$ mass is comparable to $\Delta_{0}$ [24, 27], which is likely the case for all but the largest $\Delta_{0}$ that we consider. We must also consider the thermally excited pseudo-Nambu-Goldstone bosons, but we show in Section $\overline{V A}$ that their effects are negligible. The ninth Nambu-Goldstone boson, corresponding to the spontaneous breaking of $U(1)_{B}$ and hence to superfluidity, remains massless even once quark masses are taken into 
account and therefore plays a crucial role in many low energy properties of the CFL phase, for example in its specific heat, thermal conductivity, neutrino opacity, and neutrino emissivity at low temperatures [27, 28, 29]. However, because it is neutral it will play no role in our analysis.

The excitations of the CFL phase that are most relevant to our considerations are the nine fermionic quasiparticles, whose excitation requires the breaking of pairs. In analyzing the response of the CFL phase to the strange quark mass at zero temperature, the quasiquark dispersion relations signal the instability corresponding to the disruption of pairing that constitutes the $\mathrm{CFL} \rightarrow \mathrm{gCFL}$ transition [5, 6]. These dispersion relations are even more crucial at nonzero temperature, because they determine the number densities of thermally excited quasiparticles, some of which are charged and which we shall see therefore play a crucial role in the neutrality conditions. Furthermore, these dispersion relations define the gap equations that we shall solve at nonzero temperature, again as we shall see.

\section{Color and electric neutrality}

Stable bulk matter must be neutral under all gauged charges, whether they are spontaneously broken or not. Otherwise, the net charge density would create large electric fields, making the energy non-extensive. In the case of the electromagnetic gauge symmetry, this simply requires zero charge density. In the case of the color gauge symmetry, the formal requirement is that a chunk of quark matter should be a color singlet, i.e., its wavefunction should be invariant under a general color gauge transformation. Color neutrality, meaning equality in the numbers of red, green and blue quarks, is a less stringent constraint. A color singlet state is also color neutral, whereas the opposite is not necessarily true. However it has been shown that the projection of a color neutral state onto a color singlet state costs no extra free energy in the thermodynamic limit [30]. Analyzing the consequences of the requirement of color neutrality therefore suffices for our purposes.

In nature, electric and color neutrality are enforced by the dynamics of the electromagnetic and QCD gauge fields, whose zeroth components serve as chemical potentials which take on values that enforce neutrality [4, 31]. Since we are limiting ourselves to color neutrality and not color singletness we have to consider only the $U(1) \times U(1)$ diagonal subgroup of the color gauge group. This subgroup is generated by the diagonal generators $T_{3}=$ $\operatorname{diag}\left(\frac{1}{2},-\frac{1}{2}, 0\right)$ and $T_{8}=\operatorname{diag}\left(\frac{1}{3}, \frac{1}{3},-\frac{2}{3}\right)$ of the $S U(3)$ gauge group. Electromagnetism is generated by $Q=$ $\operatorname{diag}\left(\frac{2}{3},-\frac{1}{3},-\frac{1}{3}\right)$ in flavor space $(u, d, s)$. The zeroth components of the respective gauge fields serve as chemical potentials $\mu_{3}$ and $\mu_{8}$ coupled to $T_{3}$ and $T_{8}$ charges, and as an electrostatic potential $\mu_{e}$ coupled to the negative electric charge $Q$. (We make this last choice so that $\mu_{e}>0$ corresponds to a density of electrons, not positrons.) The dynamics of the gauge potentials then require that the charge densities, which are the derivatives of the free energy with respect to the chemical potentials, must vanish:

$$
\begin{aligned}
& n_{Q}=\frac{\partial \Omega}{\partial \mu_{e}}=0 \\
& n_{3}=-\frac{\partial \Omega}{\partial \mu_{3}}=0 \\
& n_{8}=-\frac{\partial \Omega}{\partial \mu_{8}}=0 .
\end{aligned}
$$

In an NJL model with fermions but no gauge fields that we shall employ, one has to introduce the chemical potentials $\mu_{e}, \mu_{3}$ and $\mu_{8}$ "by hand" in order to enforce color and electric neutrality in the same way that gauge field dynamics does in QCD [4].

A generic diquark condensate will be neither electrically nor color neutral, so it will spontaneously break these gauge symmetries. However it may be neutral under a linear combination of electromagnetism and color. Indeed, any condensate of the form (2) is neutral with respect to the "rotated electromagnetism" generated by $\tilde{Q}=Q-T_{3}-\frac{1}{2} T_{8}$, so $U(1)_{\tilde{Q}}$ is never broken. This means that the corresponding gauge boson (the " $\tilde{Q}$-photon"), a mixture of the ordinary photon and one of the gluons, remains massless. In both the CFL and gCFL phases, the rest of the $S U(3)_{\text {color }} \times U(1)_{Q}$ gauge group is spontaneously broken, meaning that the combination of the photon and gluons orthogonal to the $\tilde{Q}$-photon, and all the other gluons, become massive by the Higgs mechanism.

In the CFL phase at $T=0$, many consequences of the neutrality equations can be analyzed in a modelindependent way [4]. A central result is that the free energy $\Omega$ is independent of

$$
\mu_{\tilde{Q}}=-\frac{4}{9}\left(\mu_{e}+\mu_{3}+\frac{1}{2} \mu_{8}\right),
$$

the chemical potential for $\tilde{Q}$-charge, as long as $\mu_{\tilde{Q}}$ is not so large that it disrupts CFL pairing. This means that the CFL phase is a $\tilde{Q}$-insulator at $T=0 \underline{32}$. Translating back into $\mu_{e}, \mu_{3}$ and $\mu_{8}$, this means that two of these three can be fixed as functions of the third:

$$
\begin{aligned}
& \mu_{3}=\mu_{e}, \\
& \mu_{8}=-\frac{M_{s}^{2}}{2 \mu}+\frac{\mu_{e}}{2},
\end{aligned}
$$

where $\mu_{e}$ can lie anywhere within the range $-\Delta_{2}+\frac{M_{s}^{2}}{2 \mu}<$ $\mu_{e}<+\Delta_{3}$. If the lower (upper) bound of this range is violated $r s-b u(r d-g u)$ pairing is disrupted: this range can be thought of as the bandgap for the CFL insulator [5] . The free energy $\Omega$ has a plateau within this range of $\mu_{\tilde{Q}}$. Electrons add a new term $\sim \mu_{e}^{4}$ to $\Omega$, adding a gentle curvature to the plateau, and selecting $\mu_{e}=0$, meaning zero electron density, as the correct solution for neutral 
CFL matter at $T=0 . \quad\left(\mu_{3}\right.$ and $\mu_{8}$ are then given by (78) with $\mu_{e}=0$.) At finite temperature where quasiquarks with non-trivial $\tilde{Q}$ are excited, we shall see that because $\Omega$ has an only-very-gently-curved plateau, it will not take much thermal excitation of quasiquarks to shift $\mu_{e}$ significantly away from zero.

\section{A Model for the Thermodynamic Potential}

We are interested in physics at non-asymptotic densities, and therefore cannot use weak-coupling methods. We are interested in physics at zero temperature and high density, at which the fermion sign problem is acute and the current methods of lattice QCD can therefore not be employed. For this reason, we need to introduce a model in which the interaction between quarks is simplified, while still respecting the symmetries of QCD, and in which the effects of $M_{s}, \mu_{e}, \mu_{3}, \mu_{8}$ and $T$ on CFL pairing can all be investigated. The natural choice is to model the interactions between quarks using a point-like four-fermion interaction, which we shall take to have the quantum numbers of single-gluon exchange. We work in Euclidean space. Our partition function $\mathcal{Z}$ and free energy density $\Omega$ are then defined by

$$
\begin{aligned}
\mathcal{Z} & =\mathrm{e}^{-\beta V \Omega}=\mathcal{N} \int \mathcal{D} \bar{\psi} \mathcal{D} \psi \exp \left(\int \mathcal{L}(x) d^{4} x\right) \\
\mathcal{L}(x) & =\bar{\psi}(i \not \partial+\boldsymbol{\mu}-\boldsymbol{M}) \psi-\frac{3}{8} G\left(\bar{\psi} \Gamma_{\mu}^{A} \psi\right)\left(\bar{\psi} \Gamma_{A}^{\mu} \psi\right)
\end{aligned}
$$

where the fields live in a box of volume $V$ and Euclidean time length $\beta=1 / T$, and $\boldsymbol{\mu}=\boldsymbol{\mu} \gamma_{4}$. The interaction vertex has the color, flavor, and spin structure of the QCD gluon-quark coupling, $\Gamma_{\mu}^{A}=\gamma_{\mu} T^{A}$ with the $T_{A}$ normalized such that $\operatorname{Tr} T_{A} T_{B}=2 \delta_{A B}$. The mass matrix $\boldsymbol{M}=\operatorname{diag}\left(0,0, M_{s}\right)$ in flavor space. The chemical potential $\boldsymbol{\mu}$ is a diagonal color-flavor matrix depending on $\mu, \mu_{e}, \mu_{3}$ and $\mu_{8}$. The normalization of the fourfermion coupling $3 G / 8$ is as in the first paper in Ref. [1]. In real QCD, the ultraviolet modes decouple because of asymptotic freedom but, in the NJL model, we have to add this feature by hand, through a UV momentum cutoff $\Lambda$ in the momentum integrals. The model therefore has two parameters, the four-fermion coupling $G$ and the three-momentum cutoff $\Lambda$, but it is more useful to parameterize the interaction in terms of a physical quantity, namely the CFL gap parameter at $M_{s}=T=0$ at a reference chemical potential that we shall take to be $500 \mathrm{MeV}$. We shall call this reference gap $\Delta_{0}$. We have checked that if we vary the cutoff $\Lambda$ by $20 \%$ while simultaneously varying the bare coupling $G$ so as to keep $\Delta_{0}$ fixed, then our results change by a few percent at most. All the results that we present are for $\Lambda=800 \mathrm{MeV}$. Most of the results that we present are for a coupling strength chosen such that $\Delta_{0}=25 \mathrm{MeV}$, but we shall also discuss results obtained with stronger couplings for which $\Delta_{0}=40 \mathrm{MeV}$ and $\Delta_{0}=100 \mathrm{MeV}$.
The free energy $\Omega$ obtained from the Lagrangian (91) upon making the ansatz (2) for the diquark condensate and working in the mean field approximation has been presented in Ref. 6]. More sophisticated derivations exist in the literature 1], but since we are assuming that the only condensate is of the form (2) we can Fierz transform the interaction to yield products of terms that appear in (2), and discard all the other terms that arise in the Fierz transformed interaction which would anyway vanish after making the mean field approximation. This yields

$$
\mathcal{L}_{\text {int }}=\frac{G}{4} \sum_{\eta}\left(\bar{\psi} P_{\eta} \bar{\psi}^{T}\right)\left(\psi^{T} \bar{P}_{\eta} \psi\right)
$$

where

$$
\left.\left(P_{\eta}\right)_{i j}^{\alpha \beta}=C \gamma_{5} \epsilon^{\alpha \beta \eta} \epsilon_{i j \eta} \quad \text { (no sum over } \eta\right)
$$

and $\bar{P}_{\eta}=\gamma_{4} P_{\eta}^{\dagger} \gamma_{4}$. The index $\eta$ labels the pairing channel: $\eta=1,2$, and 3 correspond to $d-s$ pairing, $u$-s pairing, and $u-d$ pairing. The gap parameters can then be defined precisely as

$$
\Delta_{\eta}=\frac{1}{2} G\left\langle\psi^{\mathrm{T}} \bar{P}_{\eta} \psi\right\rangle .
$$

As derived in Ref. [6], the free energy can then be written as

$$
\begin{aligned}
\Omega & =-\frac{1}{4 \pi^{2}} \int_{0}^{\Lambda} d p p^{2} \sum_{j}\left\{\left|\varepsilon_{j}(p)\right|+2 T \ln \left(1+\mathrm{e}^{-\left|\varepsilon_{j}(p)\right| / T}\right)\right\} \\
& +\frac{1}{G}\left(\Delta_{1}^{2}+\Delta_{2}^{2}+\Delta_{3}^{2}\right)-\frac{\mu_{e}^{4}}{12 \pi^{2}}-\frac{\mu_{e}^{2} T^{2}}{6}-\frac{7 \pi^{2} T^{4}}{180}, \quad \text { (13) }
\end{aligned}
$$

where the electron contribution for $T \neq 0$ has been included. The functions $\varepsilon_{j}(p)$ are the dispersion relations for the fermionic quasiparticles. They are not explicitly $T$-dependent, but they do depend on the $\Delta$ 's and $\mu$ 's which are $T$-dependent. The quasiquark dispersion relations $\varepsilon_{j}(p)$ are the values of the energy at which the propagator diverges,

$$
\operatorname{det} S^{-1}\left(-i \varepsilon_{j}(p), p\right)=0 .
$$

Here, the inverse propagator $S^{-1}$ is the $72 \times 72$ matrix

$$
S^{-1}(p)=\left(\begin{array}{cc}
\not p+\mu-M & P_{\eta} \Delta_{\eta} \\
\bar{P}_{\eta} \Delta_{\eta} & (\not p-\mu+\boldsymbol{M})^{T}
\end{array}\right)
$$

that acts on Nambu-Gor'kov spinors

$$
\Psi=\left(\begin{array}{c}
\psi(p) \\
\bar{\psi}^{T}(-p)
\end{array}\right), \quad \bar{\Psi}=\left(\bar{\psi}(p) \psi^{T}(-p)\right) .
$$

The sum in (13) is understood to run over 36 roots, because the Nambu-Gor'kov formalism has artificially made each $\left|\varepsilon_{j}\right|$ doubly degenerate. There is a further (physical) degeneracy coming from spin, meaning that there are only 18 distinct dispersion relations to be found. The free energy of any solution to the gap equations obtained 
from this mean field free energy is the same as that obtained from the Cornwall-Jackiw-Tomboulis effective potential [33], with the mean field approximation implemented via dropping the contribution of $2 \mathrm{PI}$ diagrams.

We shall evaluate $\Omega$ numerically, meaning that at every value of the momentum $p$ we must find the zeros of the inverse propagator. In order to do this numerically, we follow Ref. 11] and note that it is equivalent and faster to find the eigenvalues of the "Dirac Hamiltonian density" $\mathcal{H}(p)$, which is related to $S^{-1}$ by

$$
\operatorname{det} S^{-1}\left(p_{4}, p\right)=\operatorname{det}\left[\gamma^{4}\left(i p_{4}-\mathcal{H}(p)\right)\right],
$$

which makes it clear that eigenvalues of $\mathcal{H}(p)$ are zeros of $S^{-1}$. Like $S^{-1}$, the Dirac Hamiltonian $\mathcal{H}(p)$ is a $72 \times 72$ matrix in color, flavor, spin, and Nambu-Gor'kov space. This matrix can be decomposed into 4 blocks - three $16 \times 16$ matrices for the $(r d-g u),(r s-b u)$ and $(g s-b d)$ pairing sectors, and one $24 \times 24$ matrix for the (ru-gd-bs) sector. The absolute values of the eigenvalues are quadruply degenerate due to the Nambu-Gor'kov doubling and the spin degeneracy.

We have found considerably simpler expressions for the $(r d-g u),(r s-b u)$ and $(g s-b d)$ sectors. The calculation becomes easier if one adopts a different choice of NambuGor'kov basis, i.e. $\Psi=\left(\psi(p), C \bar{\psi}^{\mathrm{T}}(-p)\right)^{\mathrm{T}}$ as in Ref. [34]. Then the Nambu-Gor'kov and spin degeneracies are manifest, as the $16 \times 16$ matrix separates into $4 \times 4$ blocks with the form

$$
\mathcal{H}_{(g s-b d)}=\left(\begin{array}{cccc}
-\mu_{b d} & p & 0 & i \Delta_{1} \\
p & -\mu_{b d} & -i \Delta_{1} & 0 \\
0 & i \Delta_{1} & \mu_{g s}+M_{s} & p \\
-i \Delta_{1} & 0 & p & \mu_{g s}-M_{s}
\end{array}\right) \text { (18) }
$$

for the $(g s-b d)$ sector and likewise for other sectors. Each of the 4 eigenvalues of this matrix, and of its counterparts for the other sectors, contributes twice (for spin) in the sum in (13). The quark chemical potentials occurring in (18) and below are defined straightforwardly:

$$
\begin{aligned}
\mu_{r u} & =\mu-\frac{2}{3} \mu_{e}+\frac{1}{2} \mu_{3}+\frac{1}{3} \mu_{8}, \\
\mu_{g d} & =\mu+\frac{1}{3} \mu_{e}-\frac{1}{2} \mu_{3}+\frac{1}{3} \mu_{8}, \\
\mu_{b s} & =\mu+\frac{1}{3} \mu_{e}-\frac{2}{3} \mu_{8}, \\
\mu_{r d} & =\mu+\frac{1}{3} \mu_{e}+\frac{1}{2} \mu_{3}+\frac{1}{3} \mu_{8}, \\
\mu_{g u} & =\mu-\frac{2}{3} \mu_{e}-\frac{1}{2} \mu_{3}+\frac{1}{3} \mu_{8}, \\
\mu_{r s} & =\mu+\frac{1}{3} \mu_{e}+\frac{1}{2} \mu_{3}+\frac{1}{3} \mu_{8}, \\
\mu_{b u} & =\mu-\frac{2}{3} \mu_{e}-\frac{2}{3} \mu_{8}, \\
\mu_{g s} & =\mu+\frac{1}{3} \mu_{e}-\frac{1}{2} \mu_{3}+\frac{1}{3} \mu_{8}, \\
\mu_{b d} & =\mu+\frac{1}{3} \mu_{e}-\frac{2}{3} \mu_{8} .
\end{aligned}
$$

In the $(r u-g d-b s)$ sector, we could not find any simple way to make the spin degeneracy manifest, but the Nambu-Gor'kov degeneracy is manifest as the $24 \times 24$ matrix separates into $12 \times 12$ blocks with the form

$$
\mathcal{H}_{(r u-g d-b s)}=\left(\begin{array}{cccccccccccc}
-\mu_{r u} & p & 0 & -i \Delta_{3} & 0 & -i \Delta_{2} & 0 & 0 & 0 & 0 & 0 & 0 \\
p & -\mu_{r u} & i \Delta_{3} & 0 & i \Delta_{2} & 0 & 0 & 0 & 0 & 0 & 0 & 0 \\
0 & -i \Delta_{3} & \mu_{g d} & p & 0 & 0 & 0 & -i \Delta_{1} & 0 & 0 & 0 & 0 \\
i \Delta_{3} & 0 & p & \mu_{g d} & 0 & 0 & i \Delta_{1} & 0 & 0 & 0 & 0 & 0 \\
0 & -i \Delta_{2} & 0 & 0 & \mu_{b s}+M_{s} & p & 0 & 0 & 0 & -i \Delta_{1} & 0 & 0 \\
i \Delta_{2} & 0 & 0 & 0 & p & \mu_{b s}-M_{s} & 0 & 0 & i \Delta_{1} & 0 & 0 & 0 \\
0 & 0 & 0 & -i \Delta_{1} & 0 & 0 & -\mu_{b s}+M_{s} & p & 0 & 0 & 0 & -i \Delta_{2} \\
0 & 0 & i \Delta_{1} & 0 & 0 & 0 & p & -\mu_{b s}-M_{s} & 0 & 0 & i \Delta_{2} & 0 \\
0 & 0 & 0 & 0 & 0 & -i \Delta_{1} & 0 & 0 & -\mu_{g d} & p & 0 & -i \Delta_{3} \\
0 & 0 & 0 & 0 & i \Delta_{1} & 0 & 0 & 0 & p & -\mu_{g d} & i \Delta_{3} & 0 \\
0 & 0 & 0 & 0 & 0 & 0 & 0 & -i \Delta_{2} & 0 & -i \Delta_{3} & \mu_{r u} & p \\
0 & 0 & 0 & 0 & 0 & 0 & i \Delta_{2} & 0 & i \Delta_{3} & 0 & p & \mu_{r u}
\end{array}\right) .
$$

The 12 eigenvalues of this matrix each contribute once in the sum in (13). They occur in degenerate pairs due to spin.

A stable, neutral phase must minimize the free energy (13) with respect to variation of the three gap parameters
$\Delta_{1}, \Delta_{2}, \Delta_{3}$, meaning it must satisfy

$$
\frac{\partial \Omega}{\partial \Delta_{1}}=0, \quad \frac{\partial \Omega}{\partial \Delta_{2}}=0, \quad \frac{\partial \Omega}{\partial \Delta_{3}}=0,
$$

and it must satisfy the three neutrality conditions (5). The gap equations (21) and neutrality equations (5) form a system of six coupled integral equations with unknowns 
the three gap parameters and $\mu_{e}, \mu_{3}$ and $\mu_{8}$. We have solved these equations numerically at a grid of values of $T$ and $M_{s}^{2} / \mu$. We evaluate $\Omega$ using (13), finding the $\varepsilon_{j}$ by determining the eigenvalues of the matrices specified explicitly above. We evaluate partial derivatives of $\Omega$ using finite difference methods. (We used three and five point finite difference evaluations of derivatives, with the interval in the $\Delta$ or $\mu$ with respect to which $\Omega$ is being differentiated taken as $0.04 \mathrm{MeV}$.) We have worked entirely with $\mu=500 \mathrm{MeV}$, varying $M_{s}^{2} / \mu$ by varying $M_{s}$, and have worked at three values of the coupling $G$ chosen so that $\Delta_{0}$, the gap parameter at $M_{s}=T=0$, takes on the values $\Delta_{0}=25,40$ and $100 \mathrm{MeV}$.

\section{E. Approximations made and not made}

Having described our calculation in explicit detail, we close this section by enumerating the approximations that we are making, and stressing several that we have not made.

We have made the following approximations:

1. We work throughout in a mean field approximation. Gauge field fluctuations will surely be important, and must be included in future work.

2. We neglect contributions to the condensates that are symmetric in color and flavor: these are known to be present and small, smaller than the contributions that we include by at least an order of magnitude [2, 15, 18].

3. We choose light quark masses $M_{u}=M_{d}=0$ and we treat the constituent strange quark mass $M_{s}$ as a parameter, rather than solving for an $\langle\bar{s} s\rangle$ condensate. These approximations should be improved upon, along the lines of Refs. 11, 34]. In future studies in which the $\langle\bar{q} q\rangle$ condensates are solved for dynamically, the six-quark 't Hooft interaction induced by instantons must be included, since it introduces terms of the form $\langle\bar{s} s\rangle\left|\Delta_{3}\right|^{2}$, for example.

4. We ignore meson condensation in both the CFL phase and gCFL phases. We shall see in Section $\nabla A$ that in the CFL phase this means that we are assuming that the instanton contribution to the $K^{0}$ mass is comparable to or larger than $\Delta_{0}$ [24, 27]. Meson condensation in the gCFL phase has yet to be analyzed.

We expect that these approximations have quantitative effects, but none preclude a qualitative understanding of the phase diagram.

The authors of Refs. 5, 6] made further simplifying assumptions. They worked only to leading nontrivial order in $\Delta_{1}, \Delta_{2}, \Delta_{3}, \mu_{e}, \mu_{3}$ and $\mu_{8}$. They neglected the effects of antiparticles. And, most serious, they incorporated $M_{s}$ only via its leading effect, namely as an effective shift $-M_{s}^{2} / \mu$ in the chemical potentials of the strange quarks. This limits the regime of applicability of their results to $M_{s} \ll \mu$. For $\Delta_{0} \sim 100 \mathrm{MeV}$, there is pairing even at $M_{s} \gtrsim \mu$ and our calculation runs into no difficulties in this regime. (We shall show some results, even though this likely corresponds to such small $\mu$ that in reality the hadronic phase has taken over.) In the next section, we shall present our $T=0$ results. Those with $\Delta_{0}=25 \mathrm{MeV}$, as in Refs. [5, 6], are in very good agreement with the results of Refs. [5, 6]. This provides quantitative evidence for the validity of the approximations made in Refs. [5, [6] at $\Delta_{0}=25 \mathrm{MeV}$.

\section{ZERO TEMPERATURE RESULTS}

In this section we shall present the results of our model analysis at $T=0$. Here and throughout this paper, we set $\mu=500 \mathrm{MeV}$ corresponding to a baryon density about ten times that in nuclear matter, which is within the range of expectations for the density at the center of compact stars. In reality, both $M_{s}$ and $\Delta_{0}$ vary with $\mu$, and our knowledge of both is uncertain. Our model is by construction well-suited to study the disruptive effects of $M_{s}$ on pairing but it is not adequate to fix the densitydependent values of either $M_{s}$ or $\Delta_{0}$ quantitatively. In this study, therefore, as in Refs. [4, 5, 6, 15] we treat both $M_{s}$ and $\Delta_{0}$ simply as parameters. We shall plot our results versus $M_{s}^{2} / \mu$, rather than versus $M_{s}$ itself, because the strength of the disruptive effects introduced by the strange quark mass are characterized by $M_{s}^{2} / \mu$. For example, the splitting between Fermi surfaces in unpaired quark matter is of order $M_{s}^{2} / 4 \mu$, and the CFL $\rightarrow$ gCFL transition occurs where $M_{s}^{2} / \mu \simeq 2 \Delta_{1}$. Although we only present results at a single value of $\mu$, because the effects of $M_{s}$ are controlled by $M_{s}^{2} / \mu$, and because in reality $M_{s}$ is itself a decreasing function of $\mu$, one can think of our plots as giving a qualitative description of the effects of varying $\mu$, with high density at small $M_{s}^{2} / \mu$ and low density at large $M_{s}^{2} / \mu$. This description is only qualitative because our plots are each made with some fixed value of $\Delta_{0}$, whereas in reality $\Delta_{0}$ depends on $\mu$.

We begin by choosing the four fermion coupling $G$ so that $\Delta_{0}=25 \mathrm{MeV}$, as in Refs. [5, 6]. The solid curves in Fig. 2] show the gap parameters as a function of $M_{s}^{2} / \mu$, and those in Fig. 3 show the chemical potentials. These plots are in very good agreement with those of Refs. [5, 6], indicating that the approximations made in that paper that we have dispensed with here, chiefly the small- $M_{s}^{2} / \mu^{2}$ assumption, were good approximations for $\Delta_{0}=25 \mathrm{MeV}$. For small $M_{s}^{2} / \mu$, we see the CFL phase with $\Delta_{1}=\Delta_{2} \simeq \Delta_{3}$. The small difference between $\Delta_{1}=\Delta_{2}$ and $\Delta_{3}$, less than $2 \%$ everywhere within the CFL phase, is an example of an effect that we can see but that cannot be seen in the small- $M_{s}^{2} / \mu^{2}$ approximation in which $M_{s}$ is approximated as a shift in the chemical potential for the strange quarks [5, 6]. The CFL $\rightarrow$ gCFL transition occurs at $M_{s}^{2} / \mu=46.8 \mathrm{MeV}$, which is very close to $2 \Delta_{1}$, since $\Delta_{1}=23.2 \mathrm{MeV}$ at 


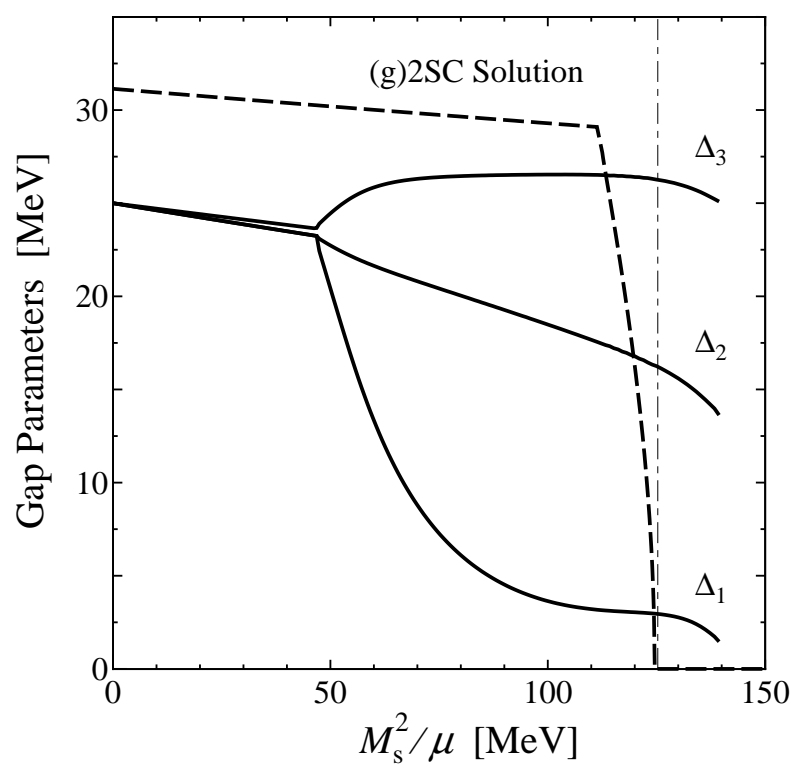

FIG. 2: Gap parameters $\Delta_{1}, \Delta_{2}$ and $\Delta_{3}$ as a function of $M_{s}^{2} / \mu$ at $T=0$. The solid curves show the CFL/gCFL solution, with the $\mathrm{CFL} \rightarrow \mathrm{gCFL}$ transition occurring where $M_{s}^{2} / \mu \simeq 2 \Delta_{1}$. There is a first order phase transition between the gCFL phase and unpaired quark matter at $M_{s}^{2} / \mu=125.3 \mathrm{MeV}$, denoted by the the thin vertical line. To the right of this line, the gCFL solution is metastable. We also find a neutral 2SC solution, with $\Delta_{3}$ given by the dashed curve in the figure, which undergoes a transition to the gapless 2SC phase of Refs. 35 at $M_{s}^{2} / \mu=112 \mathrm{MeV}$. However, from Fig. [4 we see that the (g)2SC solution is everywhere metastable, having a larger free energy than the $(\mathrm{g}) \mathrm{CFL}$ solution at the same $M_{s}^{2} / \mu$.

$M_{s}^{2} / \mu=46.8 \mathrm{MeV}$. We see from Fig. [3 that $\mu_{e} \neq 0$ in the gCFL phase, indicating a nonzero electron density. As discussed in detail in Refs. [5, [6], the negative $\tilde{Q}$-charge of the electrons is balanced by that of unpaired bu quarks, which have $\tilde{Q}=+1$, occurring in a narrow shell in momentum space. There is a larger shell in momentum space, whose width grows with increasing $M_{s}^{2} / \mu$ in the gCFL phase, within which there are unpaired $b d-$ quarks. This "blocking region" of momentum space does not contribute in the $\Delta_{1}$ gap equation, and $\Delta_{1}$ is consequently driven down. The gap equations and neutrality conditions are all coupled, and the consequences of the reduction in $\Delta_{1}$ and the increase in $\mu_{e}$ are manifest in all the curves in Figs. 2 and 3 .

As $M_{s}^{2} / \mu$ increases further, the gCFL solution eventually ceases to exist at $M_{s}^{2} / \mu=139 \mathrm{MeV}$. The gCFL solution to the gap equations is a minimum of $\Omega$ with respect to variation of the $\Delta$ 's for $M_{s}^{2} / \mu<139 \mathrm{MeV}$, becomes an inflection point at $M_{s}^{2} / \mu=139 \mathrm{MeV}$, and for larger $M_{s}^{2} / \mu$ there is no such solution. This is analyzed in greater detail in Refs. [5, [6] . The fact that the gCFL solution disappears indicates that there should be some other minimum with lower free energy, and indeed as shown in Fig. 4 we find that a first-order phase transition at which the gCFL free energy crosses above that of un-

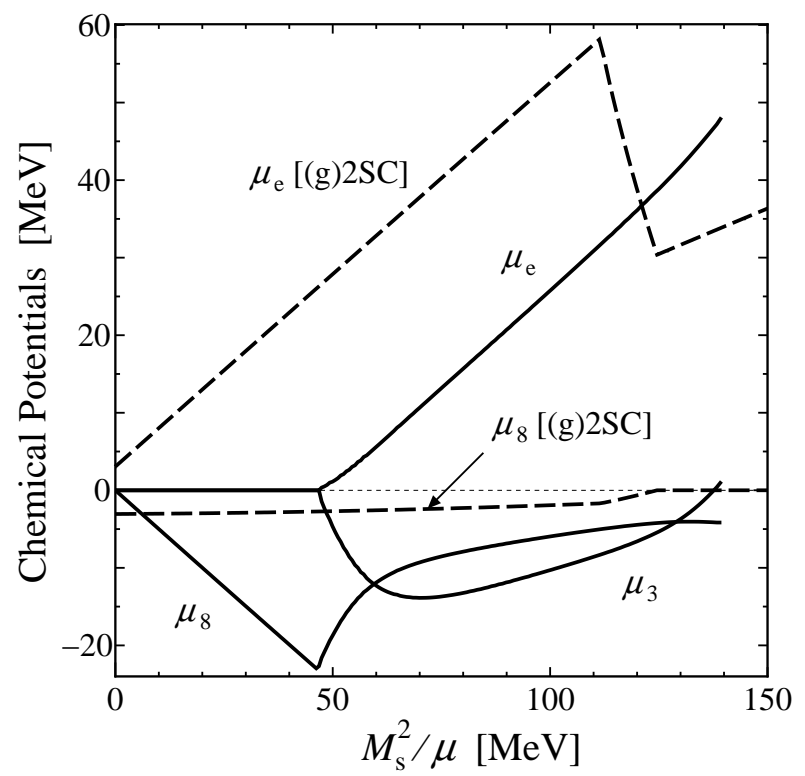

FIG. 3: Chemical potentials $\mu_{e}, \mu_{3}$ and $\mu_{8}$ as a function of $M_{s}^{2} / \mu$ at $T=0$, for the (g)CFL and (g)2SC solutions of Fig. 2 $\left(\mu_{3}=0\right.$ in the $(\mathrm{g}) 2 \mathrm{SC}$ solution and so is not shown.) Beyond the $M_{s}^{2} / \mu$ at which the g2SC solution ends, the dashed curve shows the chemical potentials $\left(\mu_{e} \neq 0\right.$ and $\left.\mu_{8}=0\right)$ for neutral unpaired quark matter.

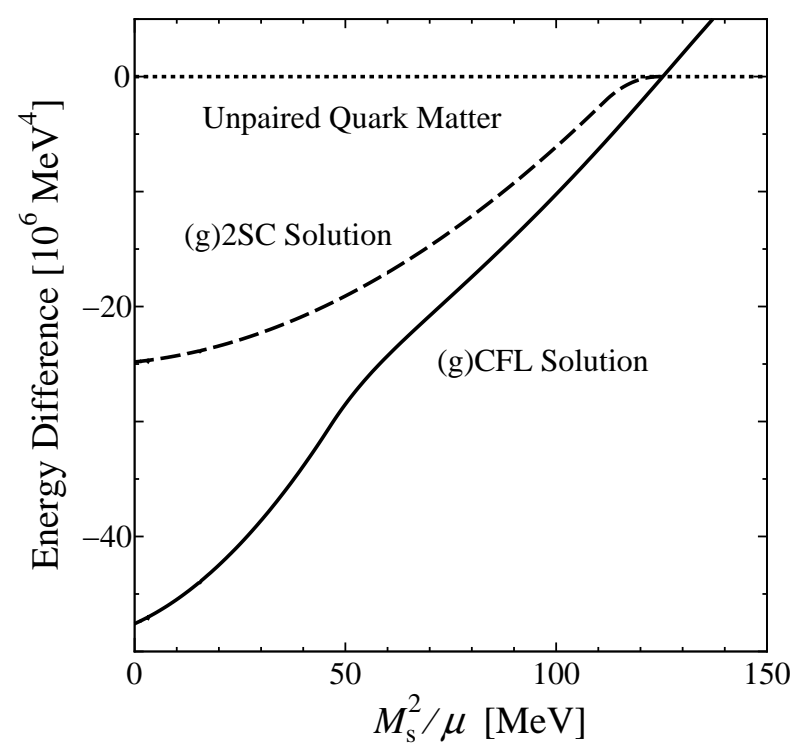

FIG. 4: Free energies of the (g)CFL and (g)2SC solutions of Fig. 2] at $T=0$, relative to that of neutral unpaired quark matter.

paired quark matter has occurred at $M_{s}^{2} / \mu=125 \mathrm{MeV}$, indicated in Fig. [2 by the vertical line. In Ref. [6], the first order phase transition and the termination of the gCFL phase at a point of inflection of the free energy occur at $M_{s}^{2} / \mu=130 \mathrm{MeV}$ and $M_{s}^{2} / \mu=144 \mathrm{MeV}$ respectively. Therefore, the errors in these quantities introduced by the small- $M_{s}^{2} / \mu^{2}$ approximation, used in Refs. [5, [6] but 
not here, are about $4 \%$ at these values of $M_{s}^{2} / \mu$.

As in Ref. [6], we find an additional neutral 2SC solution, whose gap parameter $\Delta_{3}$ and free energy are shown in Figs. 2] and 4. At $M_{s}=0$, the 2SC gap is $2^{1 / 3} \Delta_{0}$ [1, 22]. For $M_{s}^{2} / \mu$ below the $2 \mathrm{SC} \rightarrow \mathrm{g} 2 \mathrm{SC}$ transition at $M_{s}^{2} / \mu=112 \mathrm{MeV}, r d-g u$ and $r u-g d$ pairing occur at all momenta; above this transition, in the gapless 2SC phase [35, 36], there is a blocking region [37] in momentum space in which one finds unpaired $r d$ and $g d$ quarks, and $\Delta_{3}$ drops precipitously. An analogue of the g2SC phase [38] (and in fact an analogue of the gCFL phase [39]) were first analyzed in contexts in which they were metastable, but it was shown in Ref. 35] that the g2SC phase could be stabilized in two-flavor quark matter by the constraints of neutrality. However, we see in Fig. 4 that in this three-flavor quark matter setting, the (g)2SC solution everywhere has a larger free energy than the (g)CFL solution at the same $M_{s}^{2} / \mu$, and is therefore metastable. The value of $M_{s}^{2} / \mu$ at which $\Delta_{3} \rightarrow 0$ in the g2SC solution is less than $1 \mathrm{MeV}$ below the $M_{s}^{2} / \mu$ at which the gCFL phase becomes metastable. In contrast, in Ref. [6] the g2SC solution persists to an $M_{s}^{2} / \mu$ that is less than $1 \mathrm{MeV}$ above that at which the gCFL phase free energy crosses that of unpaired quark matter. This is the one instance where the small- $M_{s}^{2} / \mu^{2}$ approximation made in Ref. 6] leads one (slightly) astray, as it predicts a (very narrow) $M_{s}^{2} / \mu$-window in which the g2SC phase is favored and we find no such window. However, we shall see below that the physics at values of $M_{s}^{2} / \mu$ that are this large compared to $\Delta_{0}$ is anyway not robust, changing qualitatively with increasing $\Delta_{0}$.

We now investigate how our zero temperature results change if we vary the strength of the coupling, and hence $\Delta_{0}$. In Fig. 5 we show the gap parameters as a function of $M_{s}^{2} / \mu$ with $\Delta_{0}=40 \mathrm{MeV}$. We have changed the scale on both the horizontal and vertical axes by the same factor of $40 / 25$. We see that the $\mathrm{CFL} \rightarrow \mathrm{gCFL}$ transition again occurs at $M_{s}^{2} / \mu \simeq 2 \Delta_{1}$, and that the shape of the curves in the gCFL region is qualitatively as before, when suitably rescaled. However, at large values of $M_{s}^{2} / \mu$ we now find a g2SC window: the gCFL free energy crosses above that of the g2SC phase - whose gap parameter $\Delta_{3}$ is also shown in Fig. [5 - at the vertical line in the figure, and the g2SC gap vanishes only at a larger $M_{s}^{2} / \mu$. (We have located the vertical line by comparing free energies, as we did in Fig. 4 but we shall not give the figure.)

If we reduce $\Delta_{0}$ from $25 \mathrm{MeV}$, rather than increasing it, and rescale both axes of Fig. 2 by the same factor by which we reduce $\Delta_{0}$, we obtain a figure that looks qualitatively like Fig. 2. We conclude that stronger interaction tends to favor a g2SC window at large values of $M_{s}^{2} / \mu$, whereas weaker interaction disfavors it. The boundary between the two cases is at $\Delta_{0}=25 \mathrm{MeV}$ in our model.

It is interesting to ask what happens at still larger $\Delta_{0}$. We show the gap parameters in our model with $\Delta_{0}=100 \mathrm{MeV}$ in Fig. 6] We see the CFL $\rightarrow$ gCFL transition at $M_{s}^{2} / \mu=2 \Delta_{1}$ once again. The physics at and

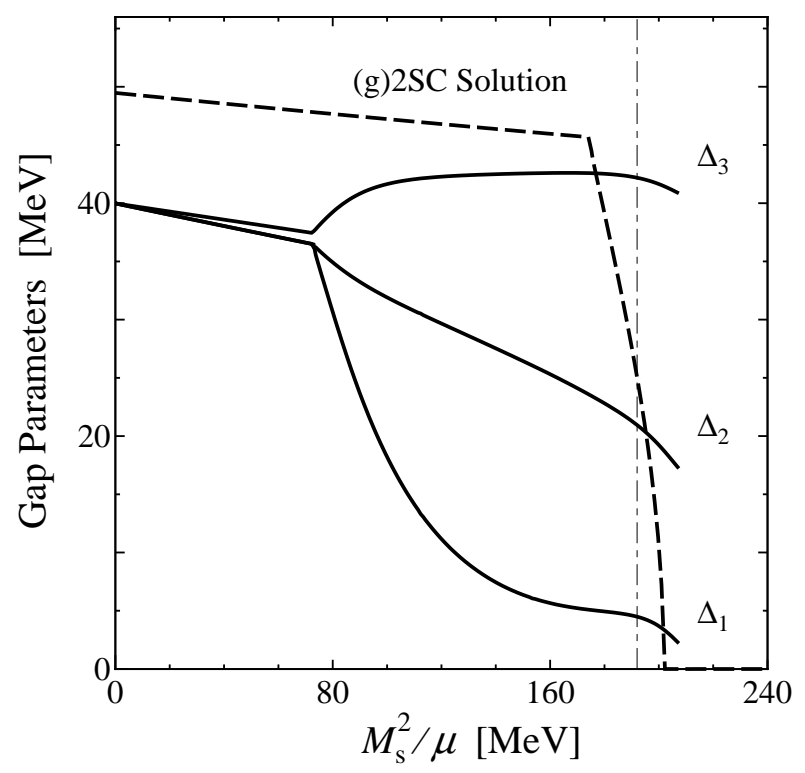

FIG. 5: Gap parameters for a stronger interaction than that in Fig. 2] chosen such that $\Delta_{0}=40 \mathrm{MeV}$ for $M_{s}=0$. As in Fig. 22 the CFL $\rightarrow$ gCFL transition occurs where $M_{s}^{2} / \mu \simeq 2 \Delta_{1}$. Here, however, the gCFL phase becomes metastable (at the thin vertical line) at a value of $M_{s}^{2} / \mu$ above which there is a g2SC solution. There is a first order $\mathrm{gCFL} \rightarrow \mathrm{g} 2 \mathrm{SC}$ transition at the thin vertical line, followed at a larger $M_{s}^{2} / \mu$ by a second order transition at which the g2SC gap $\Delta_{3}$ vanishes.

beyond the large- $M_{s}^{2} / \mu$ boundary of the gCFL regime is now qualitatively different. This regime corresponds either to very large values of $M_{s}$, or else to such small values of $\mu$ that the hadronic phase will likely have taken over, making the right half of this plot somewhat academic. One reason it is of interest, however, is simply the fact that we can draw it: had we made a small $M_{s}^{2} / \mu^{2}$ approximation as in Refs. [5, 6, 15], this regime would be inaccessible. The figure shows a sequence of phases as $M_{s}^{2} / \mu$ is increased above the gCFL phase: (i) the gCFL phase ends at a second order phase transition at which $\Delta_{1} \rightarrow 0$, above which we find a uSC window in which both $\Delta_{2}$ and $\Delta_{3}$ remain nonzero; (ii) next, $\Delta_{2} \rightarrow 0$ at a second order phase transition at which the uSC phase is succeeded by the 2SC phase; (iii) finally, there is a $2 \mathrm{SC} \rightarrow \mathrm{g} 2 \mathrm{SC}$ transition. This sequence of phases agrees with that found in Ref. 15] at large $\Delta_{0}$, in a calculation done using a small- $M_{s}^{2} / \mu^{2}$ approximation pushed beyond its regime of validity. Our present calculation can be extended (within its model context) to arbitrarily large $M_{s}$. Indeed, as can be seen in Fig. [7 we find a transition to two-flavor quark matter, with zero strange quark density, at $M_{s}=547 \mathrm{MeV}$, corresponding to $M_{s}^{2} / \mu=598 \mathrm{MeV}$. Below this $M_{s}$, we find the g2SC phase with unpaired strange quarks. Above this value of $M_{s}$, we have twoflavor g2SC quark matter and further increase in $M_{s}$ has no effect on the physics.

As $\Delta_{0}$ is increased from $40 \mathrm{MeV}$ to $100 \mathrm{MeV}$, going 


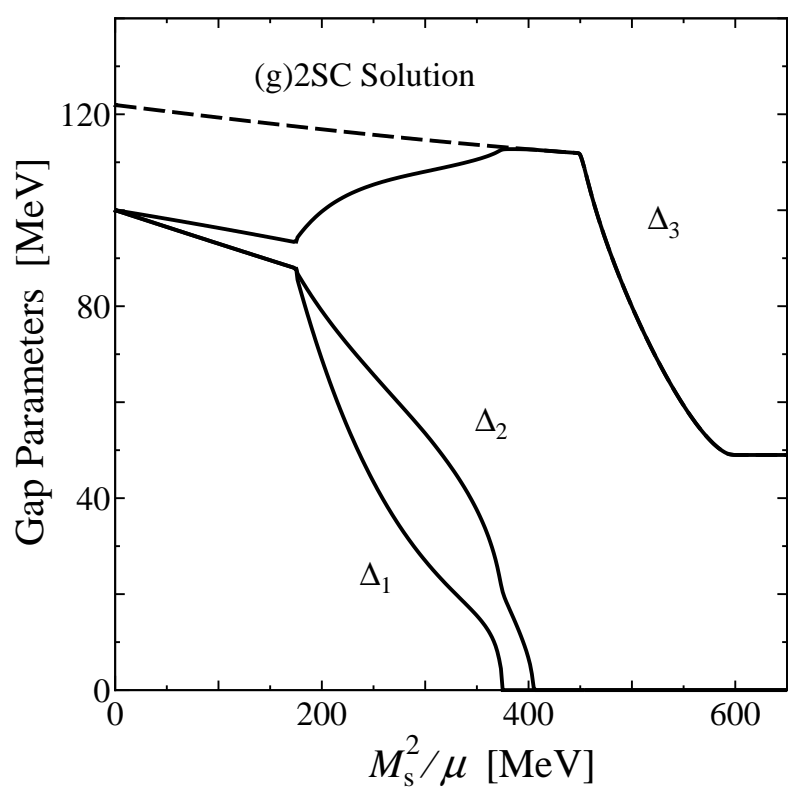

FIG. 6: Gap parameters for a still stronger interaction, with $\Delta_{0}=100 \mathrm{MeV}$ for $M_{s}=0$. As in Fig. 2 the $\mathrm{CFL} \rightarrow \mathrm{gCFL}$ transition occurs where $M_{s}^{2} / \mu \simeq 2 \Delta_{1}$. At $M_{s}^{2} / \mu=375 \mathrm{MeV}, \Delta_{1}$ vanishes at a second order $\mathrm{gCFL} \rightarrow \mathrm{uSC}$ transition. Then, at $M_{s}^{2} / \mu=405 \mathrm{MeV}, \Delta_{2}$ vanishes at a second order $\mathrm{uSC} \rightarrow 2 \mathrm{SC}$ transition. At $M_{s}^{2} / \mu=449 \mathrm{MeV}$, there is a second order $2 \mathrm{SC} \rightarrow \mathrm{g} 2 \mathrm{SC}$ transition. And finally, for $M_{s}^{2} / \mu>598 \mathrm{MeV}$, corresponding to $M_{s}>547 \mathrm{MeV}$, there are no more strange quarks present in the system, as shown explicitly in Fig. 7 and further increase in $M_{s}$ changes nothing.

from Fig. [5 to Fig. 6] the first qualitative change to occur is that the gCFL phase ends at a second order transition at which $\Delta_{1} \rightarrow 0$, instead of ending at a first order transition. Above this $\Delta_{0}$, the phase diagram includes a uSC window separated from the $(\mathrm{g}) 2 \mathrm{SC}$ phase by a first order phase transition. At a somewhat larger $\Delta_{0}$, this first order phase transition becomes second order. At a still larger $\Delta_{0}$, the interaction is strong enough to have g2SC pairing in the two flavor quark matter that our model describes for $M_{s} \rightarrow \infty$, and the physics is as in Fig. 6]

\section{THE PHASE DIAGRAM AT NONZERO TEMPERATURE}

We now explore the solutions to the gap equations and neutrality conditions at nonzero temperatures. As in the previous section, we begin with a coupling chosen so that $\Delta_{0}=25 \mathrm{MeV}$. The phase diagram for this value of the coupling is given in Fig. 11 to which the reader should refer in this section. We constructed Fig. 1 by first making plots of the gap parameters and chemical potentials versus $M_{s}^{2} / \mu$ at many values of $T$, and versus $T$ at many values of $M_{s}^{2} / \mu$. In this Section, we present and discuss several of these "sections" of Fig. 1] enough to under-

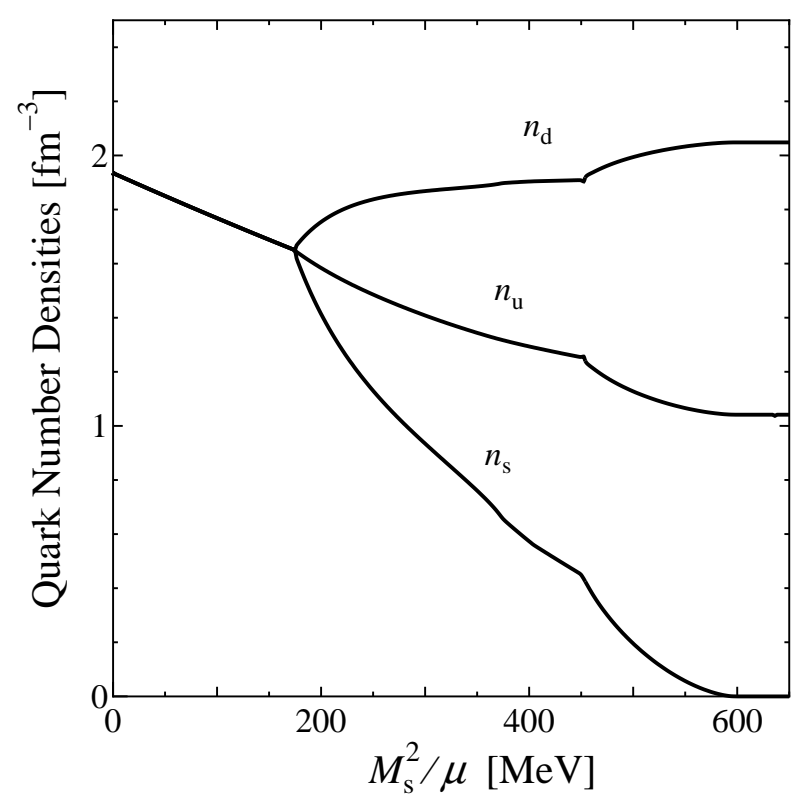

FIG. 7: Number densities of quarks with flavors $u, d$ and $s$ (in each case summed over the three colors) as a function of $M_{s}^{2} / \mu$. All parameters are as in Fig. [6] with $\Delta_{0}=100 \mathrm{MeV}$. We see that the number densities are equal only in the CFL phase, and see that for $M_{s}^{2} / \mu>598 \mathrm{MeV}$ there are no strange quarks present.

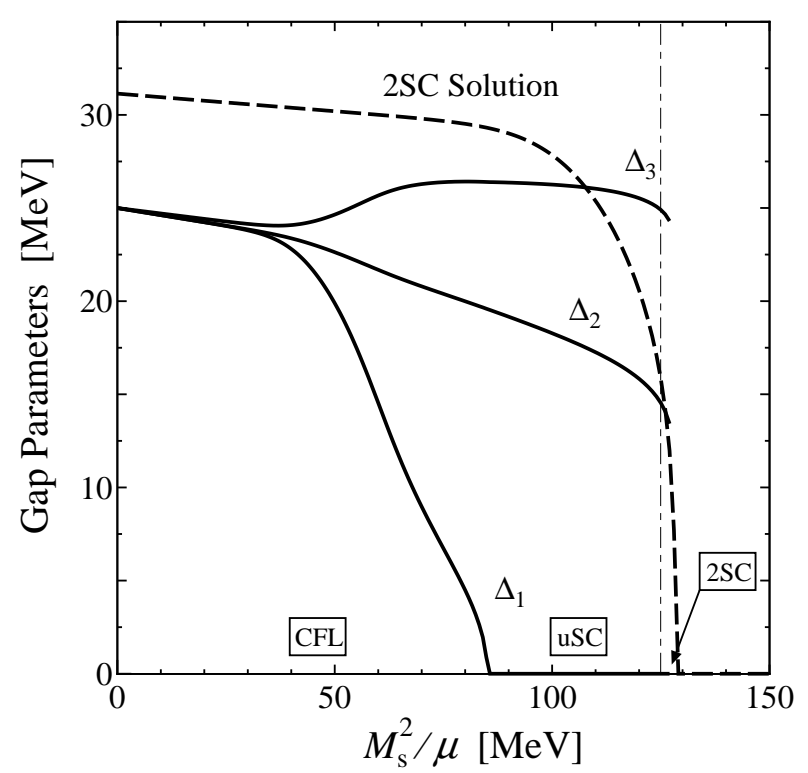

FIG. 8: Gap parameters as a function of $M_{s}^{2} / \mu$ at $T=2 \mathrm{MeV}$, with all other parameters as in Fig. 2

stand the many features of the phase diagram. We then show phase diagrams for $\Delta_{0}=40$ and $100 \mathrm{MeV}$.

We start by turning on a small temperature, $T=$ $2 \mathrm{MeV}$, and seeing how the plots of gaps and chemical potentials, shown in Figs. 8] and 9] change from those at zero temperature, Figs. 2] and 3] We see many inter- 


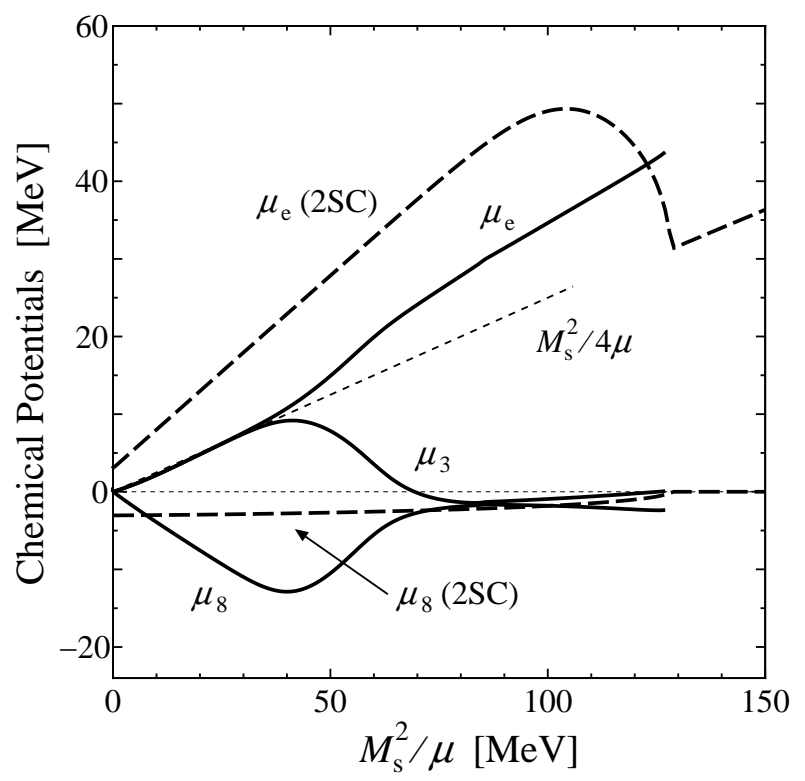

FIG. 9: Chemical potentials as a function of $M_{s}^{2} / \mu$ at $T=$ $2 \mathrm{MeV}$, with all other parameters as in Fig. 3 At small $M_{s}^{2} / \mu$, $\mu_{e}$ and $\mu_{3}$ are close to $M_{s}^{2} / 4 \mu$.

esting changes already at this relatively small temperature. The CFL $\rightarrow$ gCFL transition seen at zero temperature in Figs. 2 and 3 has become completely smooth at $T=2 \mathrm{MeV}$ : there is no sharp difference between CFL and gCFL at nonzero $T$. Furthermore, note that the zero temperature transition from 2SC to g2SC is also washed out. This makes sense: at $T=2 \mathrm{MeV}$, it makes no physical difference whether a certain fermionic quasiparticle is gapless or has a gap that is nonzero but smaller than $2 \mathrm{MeV}$. So, although in Fig. 11we have shown the values of $M_{s}^{2} / \mu$ where quark quasiparticles become gapless within the CFL, uSC and 2SC phases, these dashed lines have physical significance only where they intersect $T=0$.

We see in Fig. 8 that $\Delta_{1}$ vanishes at a second order $\mathrm{CFL} \rightarrow \mathrm{uSC}$ transition at $M_{s}^{2} / \mu=85.1 \mathrm{MeV}$. Another way to say this is that for $M_{s}^{2} / \mu<85.1 \mathrm{MeV}$, the critical temperature at which $\Delta_{1}$ vanishes upon heating must be greater than $2 \mathrm{MeV}$, whereas for $M_{s}^{2} / \mu>85.1 \mathrm{MeV}$, this critical temperature is less than $2 \mathrm{MeV}$. As at $T=0$, there is a first order phase transition, denoted in Fig. 8 by a thin vertical line, but here it is a first order phase transition between the uSC phase at $M_{s}^{2} / \mu<125 \mathrm{MeV}$ and the 2SC phase at $M_{s}^{2} / \mu>125 \mathrm{MeV}$. The 2SC phase ends at a second order phase transition where $\Delta_{3} \rightarrow 0$ at $M_{s}^{2} / \mu=129 \mathrm{MeV}$. This means that there is a regime of $M_{s}^{2} / \mu$ at which there is $2 \mathrm{SC}$ pairing at $T=2 \mathrm{MeV}$, but no pairing at $T=0$. We investigate this further below.

We see in Fig. 9] that at small $M_{s}^{2} / \mu$, the chemical potentials $\mu_{e}$ and $\mu_{3}$ are both close to $M_{s}^{2} / 4 \mu$ at $T=2 \mathrm{MeV}$. This is qualitatively different than their zero temperature behavior $\mu_{e}=\mu_{3}=0$. In comparison, $\mu_{e}$ takes on the value $M_{s}^{2} / 4 \mu$ in unpaired quark matter. Thus, already at a temperature of only $2 \mathrm{MeV}$ there is no

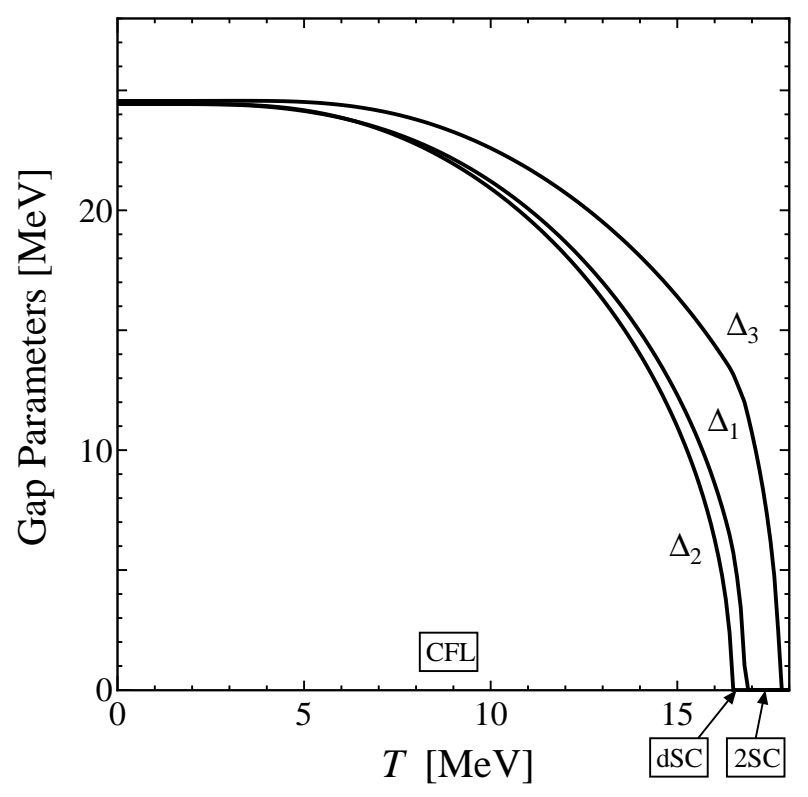

FIG. 10: Gap parameters as a function of $T$ at $M_{s}^{2} / \mu=$ $15 \mathrm{MeV}$.

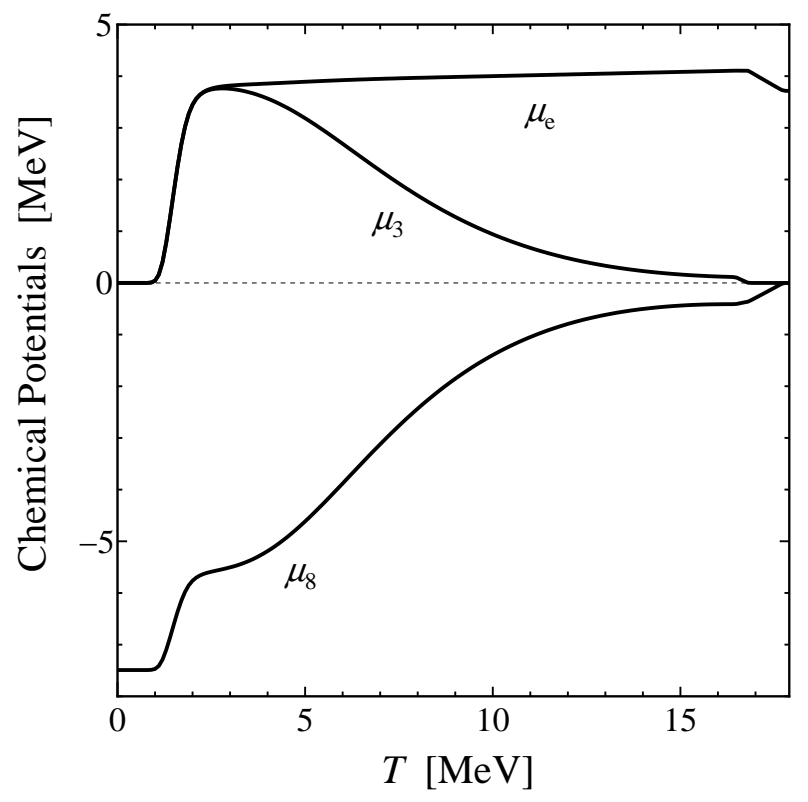

FIG. 11: Chemical potentials as a function of $T$ at $M_{s}^{2} / \mu=$ $15 \mathrm{MeV}$.

sense in which $\mu_{e}$ is small. We see that at larger $M_{s}^{2} / \mu$, $\mu_{e}$ and $\mu_{3}$ diverge as at zero temperature, but they do so smoothly and they diverge from $M_{s}^{2} / 4 \mu$, rather than from 0. Since we have found that the CFL phase has become a metal already at $T=2 \mathrm{MeV}$, it is natural to ask at what temperature the insulator-metal crossover occurs. We answer this question at $M_{s}^{2} / \mu=15 \mathrm{MeV}$ in Figs. 10] and 11] The latter figure shows a rapid insulator to metal crossover occurring between $T=1 \mathrm{MeV}$ and $T=2 \mathrm{MeV}$, with $\mu_{e}, \mu_{3}$ and $\mu_{8}$ all changing. We 


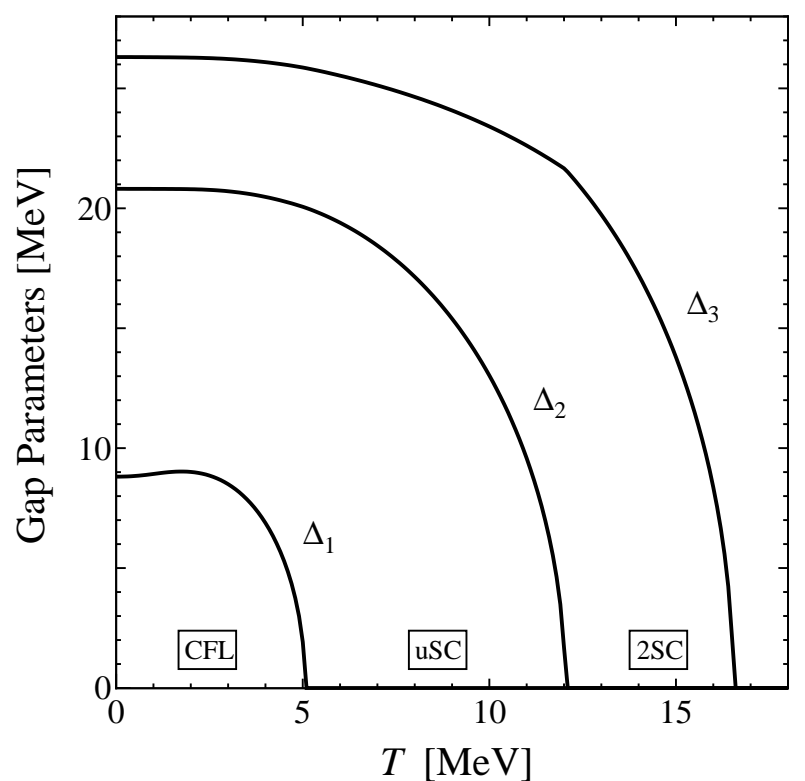

FIG. 12: Gap parameters versus temperature at $M_{s}^{2} / \mu=$ $70 \mathrm{MeV}$.

shall discuss this crossover at length in Section D. It can be understood analytically, and we shall see in Section $\nabla$ that the reason that $\mu_{e}$ takes on the value $M_{s}^{2} / 4 \mu$ is quite different from that in unpaired quark matter. Above the crossover, $\mu_{e}$ changes little as $T$ increases further but both $\mu_{3}$ and $\mu_{8}$ decrease in magnitude. This occurs because at larger temperatures the gap parameters decrease, as seen in Fig. [10] and as the gap parameters vanish color neutrality occurs with $\mu_{3}=\mu_{8}=0$ [16] whereas electrical neutrality still requires a nonzero $\mu_{e}$.

We see in Fig. 10 that the gap parameters change little at the low temperatures at which the CFL phase is undergoing its insulator to metal crossover. Although it is not really visible in the figure, we find that $\Delta_{2}>\Delta_{1}$ for $0<T<6.28 \mathrm{MeV}$, and $\Delta_{1}>\Delta_{2}$ at higher temperatures. At $T=16.46 \mathrm{MeV}, \Delta_{2}$ vanishes at a second order phase transition and we find the dSC phase. Then, at $T=16.81 \mathrm{MeV}, \Delta_{1}$ vanishes, yielding the $2 \mathrm{SC}$ phase. The final phase transition, at which $\Delta_{3}$ vanishes, occurs at $T=17.73 \mathrm{MeV}$. This ordering of phase transitions is in qualitative agreement with that found in Ref. 16 using a Ginzburg-Landau approximation, and is in disagreement with the results of Ref. [15], in which no dSC regime was found. In order to gain confidence in the accuracy of our calculation and in the existence of the dSC phase, in Section $\nabla \mathbf{D}$ we make a detailed and quantitative comparison between our results and those obtained via the Ginzburg-Landau approximation.

At larger values of $M_{s}^{2} / \mu$, the ordering of phase transitions as a function of increasing temperature changes. For example, if we consider $M_{s}^{2} / \mu=70 \mathrm{MeV}$, in the gCFL phase at $T=0$ with $\Delta_{1}<\Delta_{2}<\Delta_{3}$, we see in Fig. 12 that as the temperature is increased, $\Delta_{1}$ vanishes first, then $\Delta_{2}$ and then $\Delta_{3}$, meaning that the phase

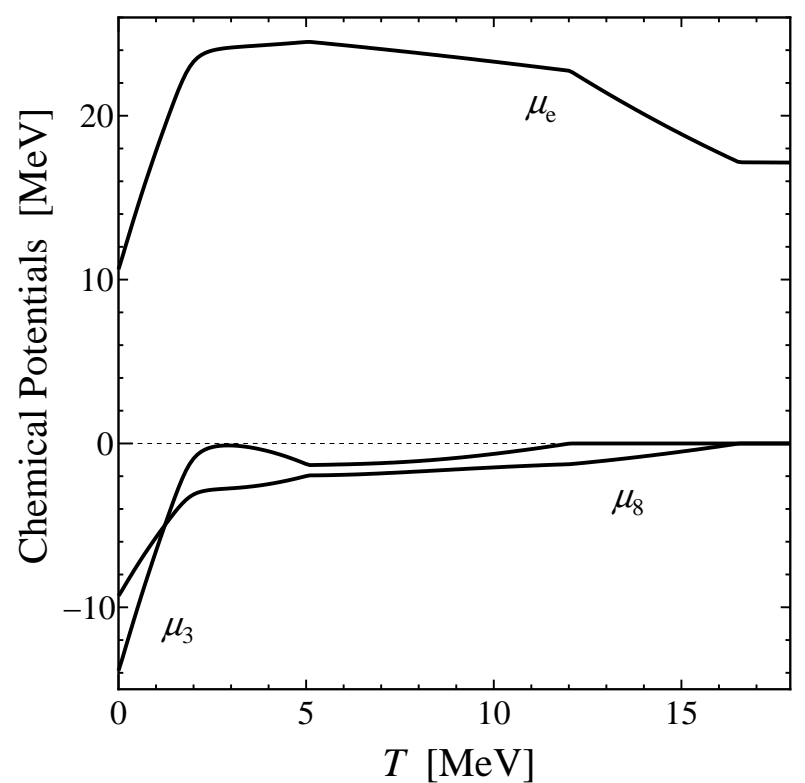

FIG. 13: Chemical potentials versus temperature at $M_{s}^{2} / \mu=$ $70 \mathrm{MeV}$.

which intervenes between CFL and 2SC is uSC, not dSC. This order of phase transitions is unsurprising, given that $\Delta_{1}<\Delta_{2}<\Delta_{3}$ at $T=0$ in the gCFL phase. All three transitions are second order transitions in mean field theory. Fig. 13 shows the chemical potentials as a function of increasing temperature at $M_{s}^{2} / \mu=70 \mathrm{MeV}$. Whereas the $T=0$ CFL phase undergoes an insulator to metal crossover as it is heated, the gCFL phase is already a metal at $T=0$.

In both Figs. 10] and [12 we see a sequence of three second order phase transitions. The first of these, a transition from the CFL phase to either the dSC or the uSC phase, is likely not significantly affected by gauge field fluctuations, because the same gauge symmetries are unbroken (the $U(1)_{\tilde{Q}}$ symmetry) and broken (the other eight gauge symmetries) on both sides of the transition. It is therefore an interesting question for future work to consider the order parameter fluctuations at this transition, asking whether they render it first order or, if not, determining its universality class. The two mean field transitions occurring at higher temperatures in Figs. 10 and 12 will be qualitatively affected by gauge field fluctuations, as at each of them there are gauge symmetries that are broken on the low temperature side of the transition and restored above the transition. Gauge field fluctuations will presumably make these transitions first order, and shift their critical temperatures upward. These effects will be significant, because the relevant gauge fields are strongly coupled [9, 10].

We have found that at small $M_{s}^{2} / \mu, \Delta_{2}$ vanishes at a lower temperature than $\Delta_{1}$ whereas at larger $M_{s}^{2} / \mu$, these two transitions occur in the opposite order. There must, therefore, be some $M_{s}^{2} / \mu$ at which both vanish at the same temperature. We see in Fig. 1 1 that this "doubly 


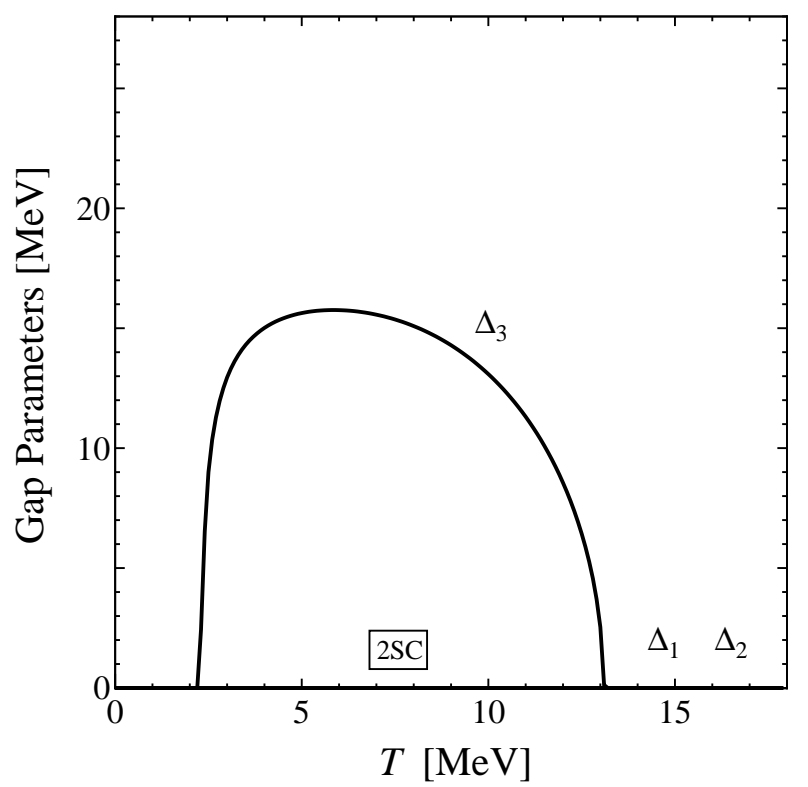

FIG. 14: Gap parameters versus temperature at $M_{s}^{2} / \mu=$ $130 \mathrm{MeV}$. At this large value of $M_{s}^{2} / \mu$, both $\Delta_{1}$ and $\Delta_{2}$ are zero at all temperatures. $\Delta_{3}=0$ at $T=0$, but this gap parameter is nonzero for $2.37 \mathrm{MeV}<T<13.08 \mathrm{MeV}$.

critical" point occurs at $T=15.3 \mathrm{MeV}$ and $M_{s}^{2} / \mu=$ $29.4 \mathrm{MeV}$.

Having followed what happens upon heating the CFL phase at $M_{s}^{2} / \mu=15 \mathrm{MeV}$ and upon heating the gCFL phase at $M_{s}^{2} / \mu=70 \mathrm{MeV}$, in Fig. 14 we consider heating quark matter at $M_{s}^{2} / \mu=130 \mathrm{MeV}$, which is unpaired at $T=0$. We see that $\Delta_{3}$ becomes nonzero at a second order phase transition, and then vanishes at a higher temperature at a second second order phase transition. This behavior has been described previously [35, 40], and can be understood as follows. At $T=0$, the $u$ and $d$ Fermi surfaces in the unpaired quark matter are too far apart to allow 2SC pairing. However, as we increase $T$, we excite $u$ quarks above the $u$ Fermi surface, and $d$ holes below the $d$ Fermi surface. This smearing of the separated Fermi surfaces assists $u$ - $d$ pairing, and $\Delta_{3}$ turns on at a nonzero temperature. Of course, at a still higher temperature the $\Delta_{3}$ condensate melts.

The final slice of the phase diagram of Fig. 1 that we shall show explicitly is a plot of the gap parameters as a function of $M_{s}^{2} / \mu$ at $T=4 \mathrm{MeV}$, shown in Fig. 15] By comparing this figure with Fig. 8 we see qualitative changes in the physics between $T=2 \mathrm{MeV}$ and $T=4 \mathrm{MeV}$ : the $2 \mathrm{SC}$ phase now takes over from the uSC phase not via a first order phase transition, but instead via a second order phase transition at $M_{s}^{2} / \mu=$ $123.2 \mathrm{MeV}$ at which $\Delta_{2} \rightarrow 0$. This means that a line of first order phase transitions, present at lower temperatures, has turned into a second order transition at a tricritical point. This tricritical point is shown by a diamond in Fig. 1] and is located between $T=3.9 \mathrm{MeV}$ and $T=4.0 \mathrm{MeV}$.

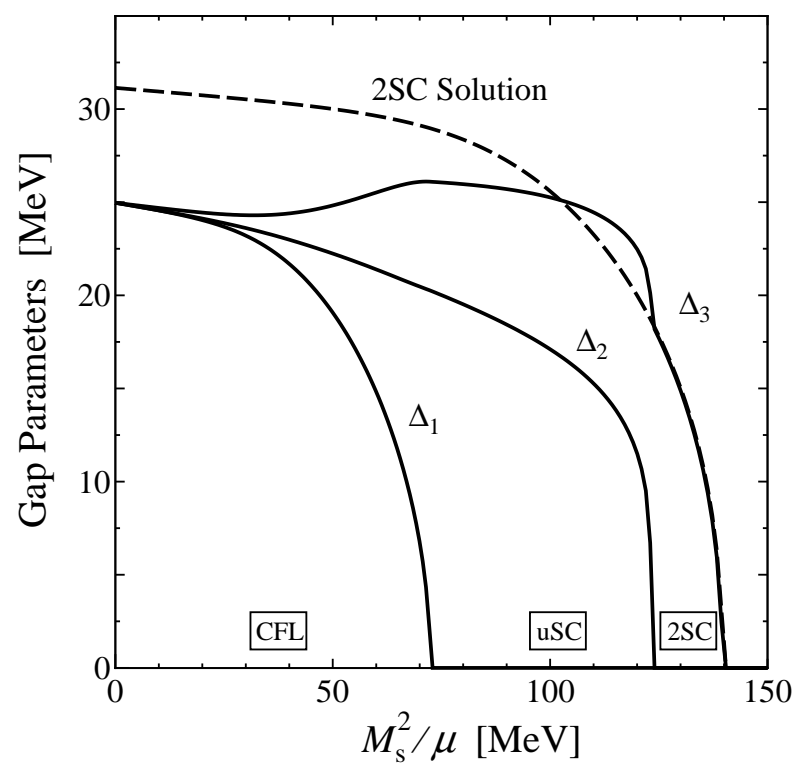

FIG. 15: Gap parameters versus $M_{s}^{2} / \mu$ at $T=4 \mathrm{MeV}$.

The results that we have presented up to this point in this section, plotted in Figs. 8 through [15] constitute a description of all of the features at $T \neq 0$ depicted in the phase diagram given in Fig. 1] We now ask how this phase diagram changes as we vary the strength of the interaction, and hence $\Delta_{0}$. If we reduce $\Delta_{0}$, there are no qualitative changes as long as we rescale the vertical and horizontal axes of the phase diagram by the same factor that we reduce $\Delta_{0}$. As at $T=0$, however, increasing $\Delta_{0}$ leads to qualitative changes in the phase diagram at large $M_{s}^{2} / \mu$, indicating that the details of the large $M_{s}^{2} / \mu$ regions of the phase diagram are not robust predictions of our model.

With $\Delta_{0}=40 \mathrm{MeV}$, in Fig. 116] we see the same three special points as in Fig. 11 a quantum critical point separating the CFL and gCFL phases at $T=0$, a doubly critical point at which the $\Delta_{1} \rightarrow 0$ and $\Delta_{2} \rightarrow 0$ transitions cross, and a tricritical point at large $M_{s}^{2} / \mu$ at which a line of first order phase transitions ends. Since both axes of Fig. 16] have been rescaled by $40 / 25$ relative to Fig. 1] the two figures are qualitatively similar: the one qualitative change occurs at large $M_{s}^{2} / \mu$, where the g2SC phase extends down to $T=0$, as we have already seen in Fig. 5. (At $T=0$, there is a sharp distinction between 2SC and g2SC, and in this instance the phase is g2SC.) The most interesting quantitative change is a change in the slopes of the $\Delta_{1} \rightarrow 0$ and $\Delta_{2} \rightarrow 0$ transitions on the phase diagram at small $M_{s}^{2} / \mu$, which pushes the doubly critical point somewhat down in temperature. This effect is more clearly visible at stronger coupling, with $\Delta_{0}=100 \mathrm{MeV}$, as shown in Fig. [17] We shall explain this quantitatively in Section VI

With $\Delta_{0}=100 \mathrm{MeV}$, in Fig. 177 we see further qualitative changes at large $M_{s}^{2} / \mu$, corresponding to those at $T=0$ shown in Fig. 6] Now, the uSC phase and 


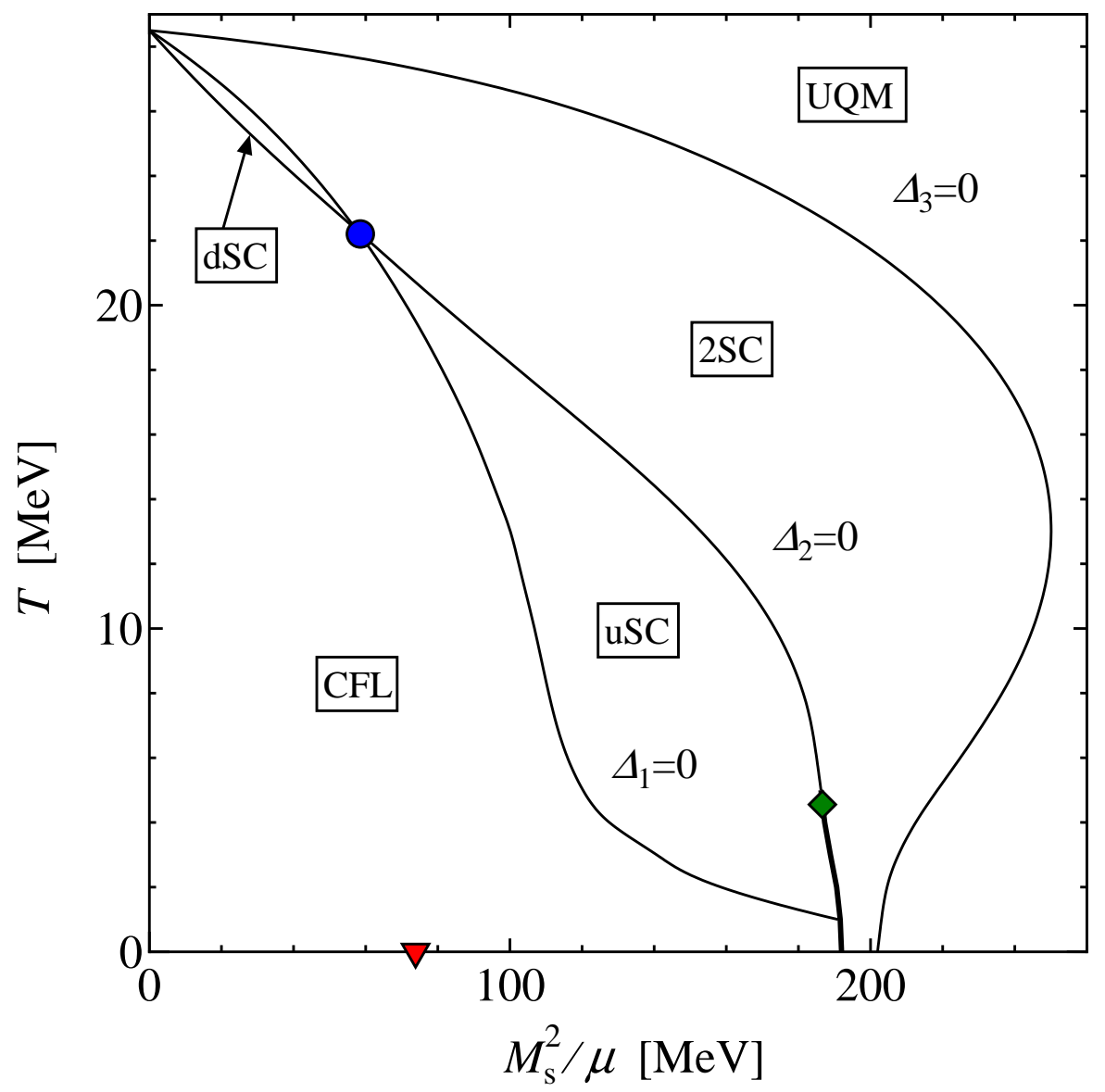

FIG. 16: Phase diagram of dense neutral quark matter in the $\left(M_{s}^{2} / \mu, T\right)$ plane, with a coupling chosen such that $\Delta_{0}=40 \mathrm{MeV}$. (All parameters except $\Delta_{0}$ are the same as in Fig. 1) Only phase transitions are shown — the analogues of the dashed and dotted lines in Fig 1 are not given.

the 2SC phase both extend to $T=0$. And, at $T=0$ there is a $2 \mathrm{SC}$ regime separated from g2SC by a quantum critical point, like that separating the CFL and gCFL phases at $T=0$. If we start at $T=0$ in the g2SC phase and heat the system, $\Delta_{3}$ at first increases, before decreasing at higher temperature, eventually vanishing at the upper phase transition shown in Fig. 17 If we extended the phase diagram to $M_{s}^{2} / \mu \rightarrow \infty$, this critical temperature would become $M_{s}$-independent in the limit. The small remaining $M_{s}$-dependence at the largest $M_{s}^{2} / \mu$ we show is easily understood: even though there are no strange quarks present at $T=0$ at these large values of $M_{s}$, meaning that the $T=0$ physics has become $M_{s}$-independent, strange quarks can still be excited at nonzero temperature.

As $\Delta_{0}$ is increased from $40 \mathrm{MeV}$ to $100 \mathrm{MeV}$, the phase diagram changes continuously from that of Fig. 16] to that of Fig. 17. First, the uSC phase reaches the $T=0$ axis. Next, the tricritical point indicated by the diamond in Fig. 16] retreats down to $T=0$. All the while, the (g)2SC region is extending farther and farther to the right, eventually to $M_{s} \rightarrow \infty$ when the coupling is strong enough to allow $u d$ pairing even once there are no strange quarks present.

We shall discuss the implications of the phase diagrams that we have found in Section VII. First, however, our investigation has raised several interesting questions that we have been able to address analytically, as we describe in Sections V and VI

\section{HEATING THE CFL PHASE: UNDERSTANDING THE INSULATOR TO METAL CROSSOVER}

We have seen in Fig. 11] that as CFL quark matter is heated, it undergoes a crossover from an insulator, with $\mu_{e}$ exponentially small, to a metal, with $\mu_{e} \sim M_{s}^{2} / 4 \mu$. With $\Delta_{0}=25 \mathrm{MeV}$ and $M_{s}^{2} / \mu=15 \mathrm{MeV}$ as in Fig. 11 the crossover occurs between $T=1 \mathrm{MeV}$ and $T=2 \mathrm{MeV}$. This insulator to metal crossover has been seen previously in Ref. [15]; our goal here is to understand it analytically.

We see from Fig. 10 that the gap parameters change little between $T=1 \mathrm{MeV}$ and $T=2 \mathrm{MeV}$, whereas the chemical potentials change dramatically. We shall 


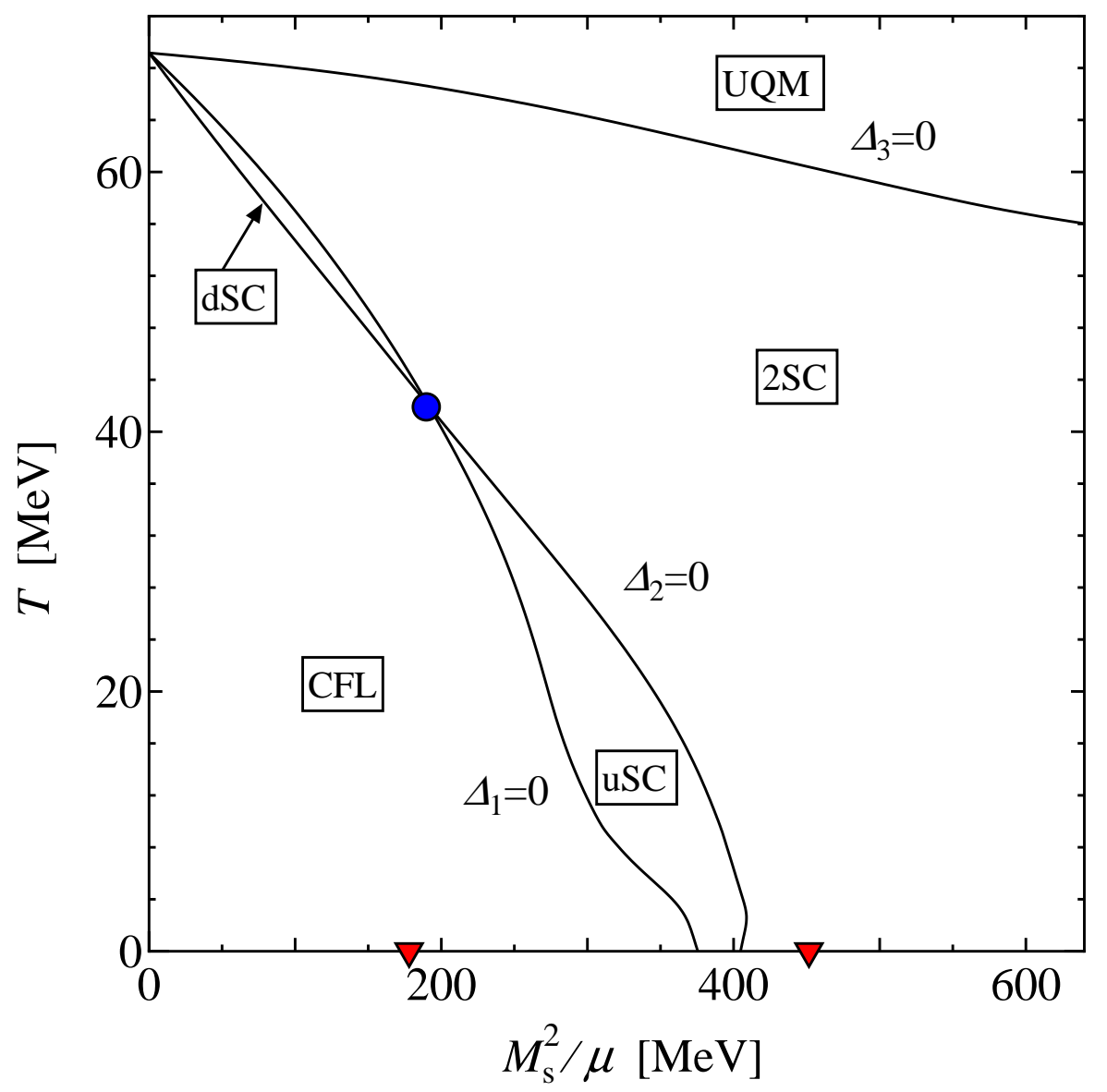

FIG. 17: Phase diagram of dense neutral quark matter in the $\left(M_{s}^{2} / \mu, T\right)$ plane, with a coupling chosen such that $\Delta_{0}=100 \mathrm{MeV}$. (All parameters except $\Delta_{0}$ are the same as in Figs. 1 and 16) The two triangles on the $T=0$ axis indicate quantum critical points at which modes become gapless. The first, which separates the CFL and gCFL phases, is familiar. The second separates 2SC and g2SC phases, as shown in Fig. 6

explain the variation of the chemical potentials, treating the gap parameters as $T$-independent. We note from Fig. 11] that during the crossover, $\mu_{e}$ increases from near 0 to near $M_{s}^{2} / 4 \mu$, while $\mu_{3}$ increases by the same amount and $\mu_{8}$ increases by half as much. This tells us that (7) and (8) are maintained, and hence it is the combination $\mu_{\tilde{Q}}$ of (6) that is changing. We recall that at $T=0$ the contribution of the quark matter to the free energy $\Omega$ is independent of $\mu_{\tilde{Q}}$, and this "plateau" is only curved by the small contribution of the electrons to the free energy, of order $\mu_{e}^{4}$, which favors $\mu_{e}=0$. Above the crossover we find $\mu_{e} \simeq M_{s}^{2} / 4 \mu$, which is on the plateau but away from the point on the plateau favored by the electron neutrality condition. In order to understand the crossover, then, there must be thermally excited $\tilde{Q}$-charged quasiparticles whose neutrality condition favors $\mu_{e} \neq 0$, and we must see the curvature of the free energy plateau due to these quasiparticles "take over" from that due to the electrons. We first show that the CFL quark quasiparticle excitations have the desired effect, and then in Section $\overline{\nabla A}$ we show that the thermally excited charged mesonic pseudo-Nambu-Goldstone boson excitations of the CFL phase play a negligible role.

The quarks with nonzero $\tilde{Q}$ are the $r s$ and $b u$ which pair with gap parameter $\Delta_{2}$, and the $r d$ and $g u$ which pair with gap parameter $\Delta_{3}$. This means that in the CFL phase there are two quasiquarks with $\tilde{Q}=+1$ (one a linear combination of bu quarks and $r s$ holes; the other a linear combination of $g u$ quarks and $r d$ holes) and two quasiquarks with $\tilde{Q}=-1$ (one a linear combination of $r s$ quarks and $b u$ holes; the other a linear combination of $r d$ quarks and $g u$ holes).

We now evaluate the dispersion relations of these excitations, and estimate the number density of thermally excited charged quasiquarks. In this Section, we shall follow Refs. 5, 6] and include the nonzero strange quark mass only via its effect as a shift in the chemical potentials of the strange quarks. We have seen in Section III that this is a good approximation in the CFL phase. The dispersion relations of these four quasiparticles are given 
by $[6]$

$$
\begin{aligned}
\varepsilon_{(r s-b u)}(p)= \pm & \frac{1}{2}\left(\mu_{r s}-\frac{M_{s}^{2}}{2 \mu}-\mu_{b u}\right) \\
& +\sqrt{\left(p-\bar{\mu}_{(b u-r s)}+\frac{M_{s}^{2}}{4 \mu}\right)^{2}+\Delta_{2}^{2}} \\
\varepsilon_{(r d-g u)}(p)= \pm & \frac{1}{2}\left(\mu_{g u}-\mu_{r d}\right)+\sqrt{\left(p-\bar{\mu}_{(r d-g u)}\right)^{2}+\Delta_{3}^{2}}
\end{aligned}
$$

where $\bar{\mu}_{b u-r s} \equiv\left(\mu_{b u}+\mu_{r s}\right) / 2$ and $\bar{\mu}_{r d-g u} \equiv\left(\mu_{r d}+\mu_{g u}\right) / 2$. Upon substituting the definitions (19) and the relations (7) and (8), which are maintained through the crossover, these become

$$
\begin{aligned}
& \varepsilon_{(r s-b u)}(p)= \pm\left(\mu_{e}-\frac{M_{s}^{2}}{2 \mu}\right)+\sqrt{\left(p-\mu+\frac{M_{s}^{2}}{6 \mu}\right)^{2}+\Delta_{2}^{2}} \\
& \varepsilon_{(r d-g u)}(p)= \pm \mu_{e}+\sqrt{\left(p-\mu+\frac{M_{s}^{2}}{6 \mu}\right)^{2}+\Delta_{3}^{2}}
\end{aligned}
$$

At the temperatures of interest, these excitation energies are all greater than $T$, and so only the lowest energy excitation with each $\tilde{Q}$ charge matters - the number density of the higher energy excitations is exponentially smaller. Labeling the quasiparticles by their $\tilde{Q}$ charge, the excitation energies of the lowest lying charged quasiquarks are

$$
\begin{aligned}
& \varepsilon_{+1}(p)=\left(\mu_{e}-\frac{M_{s}^{2}}{2 \mu}\right)+\sqrt{\left(p-\mu+\frac{M_{s}^{2}}{6 \mu}\right)^{2}+\Delta_{2}^{2}} \\
& \varepsilon_{-1}(p)=-\mu_{e}+\sqrt{\left(p-\mu+\frac{M_{s}^{2}}{6 \mu}\right)^{2}+\Delta_{3}^{2}}
\end{aligned}
$$

corresponding to the $(r s-b u)$ quasiquark with $\tilde{Q}=+1$ and the $(r d-g u)$ quasiquark with $\tilde{Q}=-1$. We now evaluate the $T$-dependent contribution of these quasiquarks to the free energy $\Omega$ given by (13). Because the quasiquark energies are much larger than the temperatures of interest, the Boltzmann factors are small, the integral is dominated by $p$ near the minimum of $\varepsilon(p)$, and we can use the saddle-point approximation. We find that, for example, the contribution of the $\tilde{Q}=+1$ quasiquark to $\Omega$ is given by

$$
\begin{aligned}
& -\frac{1}{\pi^{2}} \int_{0}^{\Lambda} d p p^{2} T \ln \left(1+\mathrm{e}^{-\left|\varepsilon_{+1}(p)\right| / T}\right) \\
\simeq & -\frac{1}{\pi^{2}} \int_{0}^{\Lambda} d p p^{2} T e^{-\left|\varepsilon_{+1}(p)\right| / T} \\
\simeq & -\frac{1}{\pi^{2}} \sqrt{2 \pi \Delta T} \bar{\mu}^{2} T e^{-\left|\varepsilon_{+1}(\bar{\mu})\right| / T}
\end{aligned}
$$

where $\bar{\mu} \equiv \mu-M_{s}^{2} / 6 \mu$. The contribution of the $\tilde{Q}=-1$ quasiparticle is analogous. The contribution of these two quasiparticles to the $\tilde{Q}$-charge density is then

$$
\begin{array}{ll}
(\tilde{Q}=+1) & \frac{\sqrt{2 \pi \Delta T} \bar{\mu}^{2}}{\pi^{2}} e^{-\left(\Delta+\mu_{e}-M_{s}^{2} / 2 \mu\right) / T}, \\
(\tilde{Q}=-1) & -\frac{\sqrt{2 \pi \Delta T} \bar{\mu}^{2}}{\pi^{2}} e^{-\left(\Delta-\mu_{e}\right) / T} .
\end{array}
$$

We have set $\Delta_{3}=\Delta_{2} \equiv \Delta$, a good approximation throughout the crossover as shown in Fig. 10] $\tilde{Q}_{-}$ neutrality is a balance between the charge densities of these two quark quasiparticles and the electrons.

We can now see that what drives the system to $\mu_{e} \neq 0$ is the fact that the lightest $\tilde{Q}=+1$ and $\tilde{Q}=-1$ quark quasiparticles have dispersion relations with different gaps when $M_{s} \neq 0$, and consequently contribute charge densities of different magnitudes when thermally excited. If we attempt to set $\mu_{e}=0$ at $T \neq 0$, there are more $\tilde{Q}=+1$ quasiparticles present than $\tilde{Q}=-1$ quasiparticles, and the system is not neutral. To achieve neutrality, $\mu_{e}$ must be increased as this increases the density of $\tilde{Q}=-1$ quasiparticles, decreases the density of $\tilde{Q}=+1$ quasiparticles, and adds electrons, which have $\tilde{Q}=-1$. This is described by the neutrality condition

$$
\begin{gathered}
\frac{2 \sqrt{2 \pi \Delta T} \bar{\mu}^{2}}{\pi^{2}} \mathrm{e}^{-\left(\Delta-M_{s}^{2} / 4 \mu\right) / T} \sinh \left(\frac{M_{s}^{2} / 4 \mu-\mu_{e}}{T}\right) \\
-\frac{\mu_{e}^{3}}{3 \pi^{2}}-\frac{\mu_{e} T^{2}}{3}=0,
\end{gathered}
$$

which we can solve for $\mu_{e}(T)$ if we take $\Delta$ to be $T$ independent.

Let us now investigate the implications of this result. At very small $T$, the quark quasiparticles are exponentially rare, and those with $\tilde{Q}=-1$ are exponentially rarer than those with $\tilde{Q}=+1$. The $\tilde{Q}=+1$ quasiparticle density is balanced by the electron density, and $\mu_{e}$ is exponentially small. However, the quasiparticle densities are proportional to $\mu^{2}$ whereas the electron density is not. This means that at the $T$ at which $\mu_{e}$ starts to take off, the quasiparticle Boltzmann factors are still rather small. Once $T$ is large enough that $\mu_{e}$ approaches $M_{s}^{2} / 4 \mu$, however, even though the individual Boltzmann factors continue to rise rapidly as $T$ increases further, the sinh factor in (28) becomes small. Neutrality at this point is primarily a balance between the densities of the $\tilde{Q}=+1$ and $\tilde{Q}=-1$ quasiparticles, with electrons cancelling only the small difference between their densities. The result, seen already in Fig. 11] and shown in greater detail in Fig. 18 is a crossover in which $\mu_{e}$ is at first exponentially small, then rises rapidly, and then saturates as it approaches $M_{s}^{2} / 4 \mu$. We see in Fig. 18 that the equation (28) for $\mu_{e}$ that we have derived, making approximations as described, gives a very good description of the numerical solution of the full coupled gap and neutrality equations. This demonstrates that (28) provides us with a good analytic description of the insulator to metal crossover that CFL quark matter experiences when heated. 


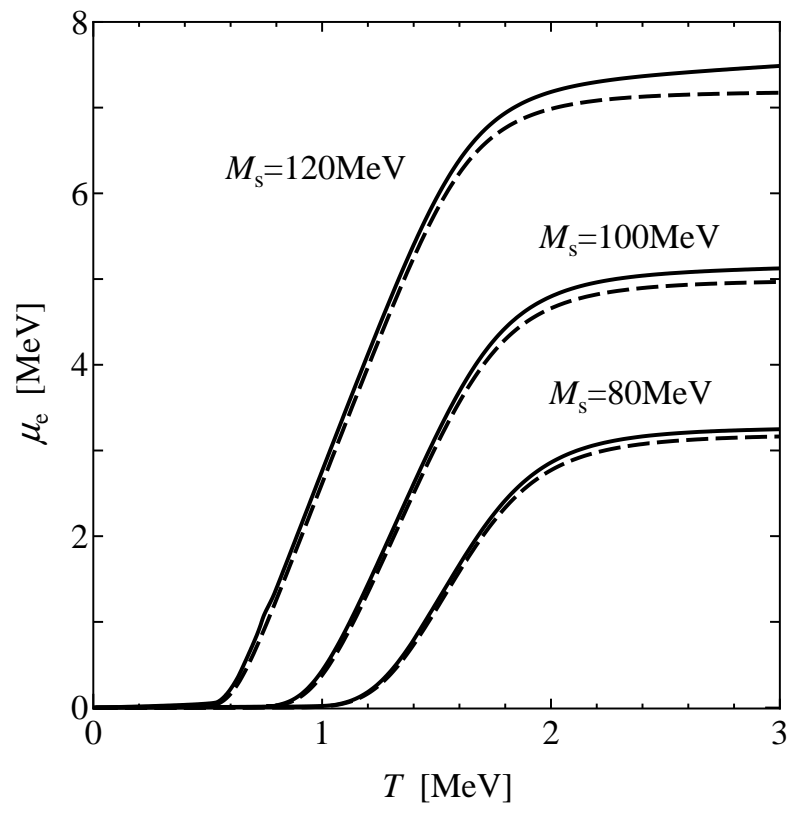

FIG. 18: Comparison between the analytic results (dashed curves) and the numerical results (solid curves) for $\mu_{e}$ as a function of temperature with $\Delta_{0}=25 \mathrm{MeV}$ for several values of $M_{s}$ in the CFL phase. The analytic results were obtained from (28), and the numerical results were obtained by solving the full coupled gap equations and neutrality conditions, as in previous Sections. In evaluating $\mu_{e}$ from (28) we have taken $\Delta$ to be the average of $\Delta_{2}$ and $\Delta_{3}$ at the midpoint of the crossover.

We have set the electron mass to zero in Fig. 18 and throughout. Including it means that it takes a larger $\mu_{e}$ to achieve a given electron density, pushing all the curves in Fig. 18 very slightly upwards. With an electron mass as in nature, the effect on the curves is invisible on the scale of the plot.

\section{A. Contribution of charged mesons}

As described in Section $\amalg B$ there are other charged excitations in the CFL phase. Among the pseudoNambu-Goldstone bosons, there are mesons with the quantum numbers of the $\pi^{ \pm}$and $K^{ \pm}$. We have neglected the contribution of thermally excited charged mesons to the charge density in the derivation of (28). We now investigate this approximation.

The dispersion relations of the charged mesons, together with that for the $K^{0}$-meson which we shall also

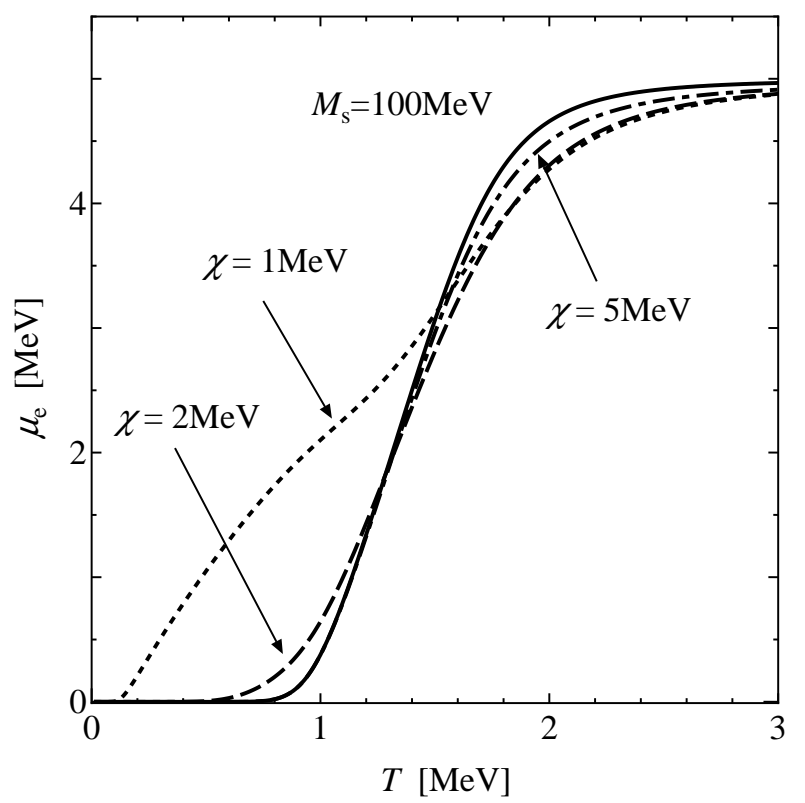

FIG. 19: Comparison between the analytic estimate for $\mu_{e}$ versus $T$ with and without charged meson contributions. The solid curve, with no meson contributions, is the same as the $M_{s}=100 \mathrm{MeV}$ analytic estimate in Fig. 18 The dashed curves include the contribution of thermally excited charged pions and kaons, for three values of the parameter $\chi$ described in the text that parameterizes the instanton contribution to the meson masses.

need below, are given by [12, 23, 24, 25, 26, 27]

$$
\begin{aligned}
& \varepsilon_{\pi^{ \pm}}(p)= \pm \mu_{e}+\sqrt{v^{2} p^{2}+M_{\pi^{ \pm}}^{2}} \\
& \varepsilon_{K^{ \pm}}(p)= \pm \mu_{e} \mp \frac{M_{s}^{2}}{2 \mu}+\sqrt{v^{2} p^{2}+M_{K^{ \pm}}^{2}} \\
& \varepsilon_{K^{0}}(p)=-\frac{M_{s}^{2}}{2 \mu}+\sqrt{v^{2} p^{2}+M_{K^{0}}^{2}},
\end{aligned}
$$

where $v^{2}=1 / 3$ at high density 25]. The meson masses in the CFL phase are given by

$$
\begin{aligned}
M_{\pi^{ \pm}}^{2} & =a\left(M_{u}+M_{d}\right) M_{s}+\chi\left(M_{u}+M_{d}\right) \\
M_{K^{ \pm}}^{2} & =a\left(M_{u}+M_{s}\right) M_{d}+\chi\left(M_{u}+M_{s}\right) \\
M_{K^{0}}^{2} & =a\left(M_{d}+M_{s}\right) M_{u}+\chi\left(M_{d}+M_{s}\right) .
\end{aligned}
$$

Here, $a=3 \Delta^{2} / \pi^{2} f_{\pi}^{2}$ with $f_{\pi}^{2}=(21-8 \log 2) \mu^{2} / 36 \pi^{2}$ at high density [25], which yields $a=0.0175$ for $\Delta_{0}=$ $25 \mathrm{MeV}$ at $\mu=500 \mathrm{MeV}$. And, $\chi$ parameterizes the contribution of $U(1)_{A}$-breaking instanton effects which generate $\langle\bar{q} q\rangle$ condensates and therefore contributions to meson masses in the CFL phase [23, 24, 27], via the 't Hooft interaction which contributes couplings of the form $\Delta^{2}\langle\bar{q} q\rangle$. The magnitude of $\chi$ is not well known, as it depends on the instanton size distribution and instanton form factors at nonzero density. It has been estimated to lie in the range $1 \mathrm{MeV}<\chi<100 \mathrm{MeV}$ [24, 27]. In Fig. 19] we include the contribution of all four charged 
mesons to the $\tilde{Q}$-charge neutrality condition, determining the density of thermally excited bosonic quasiparticles from the dispersion relations. The contribution to the charge density from the $K^{ \pm}$mesons is

$\frac{1}{2 \pi^{2}} \int_{0}^{\infty} d p p^{2}\left(\frac{1}{\exp \left[\varepsilon_{K^{+}}(p) / T\right]-1}-\frac{1}{\exp \left[\varepsilon_{K^{-}}(p) / T\right]-1}\right)$,

and that from the pions is analogous. We then solve for $\mu_{e}$ vs. $T$, taking $M_{s}=100 \mathrm{MeV}$ and $M_{u}=5 \mathrm{MeV}$ and $M_{d}=10 \mathrm{MeV}$. We plot the results for $\chi=1,2$ and 5 $\mathrm{MeV}$ in Fig. 19.

Adding the charged mesons adds new charge carriers, and so the simplest expectation for their effects is that a smaller $\mu_{e}$ will be required in order to neutralize the imbalance in the fermionic quasiparticle sector. We see in Fig. 19] that this expectation is borne out above the crossover, but not below. Above the crossover, we see that $\mu_{e}$ is reduced relative to the results we obtained in Fig. 18 where we neglected the mesons. The mesonic contributions get less significant at larger values of $\chi$, as the mesons get heavier. They are already small for $\chi=1 \mathrm{MeV}$ and are negligible by $\chi=5 \mathrm{MeV}$. At low temperatures, and for the smallest values of $\chi$, there is another effect to be understood. The dispersion relations for the $K^{ \pm}$in (29) indicate that the $K^{+}$is easier to excite than the $K^{-}$. This means that they behave like the quasiquarks, in the sense that at a nonzero temperature they contribute a positive $\tilde{Q}$-charge density, which must be cancelled. If $\chi$ is very small, the $K^{+}$charge density becomes significant at such a low temperature that its contribution can only be cancelled by electrons - the Boltzmann factors for all other excitations are still prohibitive. This means that if $\chi$ is very small, $\mu_{e}$ initially rises with temperature significantly more rapidly than in the absence of the mesons. We see this effect clearly in Fig. 19 for $\chi=1 \mathrm{MeV}$ and still to a small degree for $\chi=2 \mathrm{MeV}$. For $\chi=5 \mathrm{MeV}, \mu_{e}(T)$ is indistinguishable at low temperatures from that in the absence of the mesons.

We can summarize the results in Fig. 19] as follows. For $\chi \gtrsim 2 \mathrm{MeV}$, the thermally excited charged mesons have no significant effects at any temperature. But, if $\chi \sim 1 \mathrm{MeV}$, which is at the bottom end of the estimated allowed range $1 \mathrm{MeV}<\chi<100 \mathrm{MeV}$ [24, 27], the $K^{+}$ excitations contribute significantly at very low temperatures.

At much larger temperatures, well above the insulator to metal crossover that we have analyzed, we come to the various critical temperatures that we have seen in Section [V] and will analyze further in Section [V] At these temperatures, at which gap parameters vanish, it is well understood that the fermionic quasiparticles are the most important degrees of freedom. What we have shown is that at low temperatures also, the charged mesons are less important than the charged fermions among the thermal excitations, as long as $\chi$ is not very small.

Mesons can nevertheless play an important role if they condense [12]. At $T=0$, the CFL phase is stable against meson condensation as long as $\varepsilon_{K^{0}}>0$ at $p=0$. $\left(K^{0}\right.$ condensation yields the most stringent constraint.) This requires $M_{K^{0}}^{2}>M_{s}^{4} / 4 \mu^{2}$, corresponding to $\chi \gtrsim M_{s}^{3} / 4 \mu^{2}$. For $M_{s}=100 \mathrm{MeV}$, as in Fig. 19] this requires $\chi>$ $1 \mathrm{MeV}$. We are therefore justified in our neglect of $K^{0}$ condensation in Fig. [19] If $\chi>3.6 \mathrm{MeV}$, there is no $K^{0}$-condensation in the CFL phase with $\Delta_{0}=25 \mathrm{MeV}$ for $M_{s}^{2} / \mu<46.8 \mathrm{MeV}$, meaning that there is no $K^{0}$ condensation at $T=0$ for all values of $M_{s}^{2} / \mu$ below the $\mathrm{CFL} \rightarrow \mathrm{gCFL}$ transition. ( $K^{0}$-condensation in the gCFL phase has yet to be analyzed.) It seems likely that $\chi>$ $3.6 \mathrm{MeV}$ at accessible densities, since $\chi$ is larger at lower densities and the analysis in Ref. 24] which yields the estimate $1 \mathrm{MeV}<\chi<100 \mathrm{MeV}$ becomes more reliable at higher densities. If the coupling is stronger, however, $K^{0}$-condensation becomes more likely. For example, for $\Delta_{0}=100 \mathrm{MeV}$, there is no $K^{0}$-condensation in the CFL phase only if $\chi>26 \mathrm{MeV}$. If $K^{0}$-condensation were to occur, it delays the $\mathrm{CFL} \rightarrow \mathrm{gCFL}$ transition, increasing the $M_{s}^{2}$ at which it occurs by a factor $4 / 3$ if $\chi=0$ [13]. Further work remains to be done, for example extending the analysis of Ref. 13] to nonzero $\chi$ and to the gCFL phase.

Another issue that remains to be investigated is the possibility of $\pi^{-}$condensation at nonzero temperature. We have seen that above the insulator to metal transition, $\mu_{e} \approx M_{s}^{2} / 4 \mu$. At zero temperature, this would lead to $\pi^{-}$condensation if $\chi<M_{s}^{4} /\left(16 \mu^{2}\left(M_{u}+M_{d}\right)\right)$, but this $\mu_{e}$ only arises at $T \neq 0$, and nonzero $T$ acts to stabilize against meson condensation.

Finally, all these issues should be investigated in an expanded model in which the 't Hooft interaction is included from the beginning in the free energy and hence in the gap and neutrality conditions, and the quark masses are also solved for dynamically.

\section{THE GINZBURG-LANDAU APPROXIMATION}

In the $M_{s}^{2} / \mu \rightarrow 0$ limit, the three critical temperatures at which the gap parameters vanish become one. Near this critical temperature, where all gap parameters are small, a Ginzburg-Landau approximation can be employed. The Ginzburg-Landau free energy was analyzed at $M_{s}=0$ in Refs. [3, 17], and was extended to small but nonzero $M_{s}^{2} / \mu$ in Ref. [16]. We wish to compare our results at small but nonzero $M_{s}^{2} / \mu$ to those obtained in the Ginzburg-Landau approximation, and to compare the coefficients in the Ginzburg-Landau potential (actually, ratios of coefficients) in our model to those calculated in QCD at asymptotic densities and thus at weak coupling in Refs. [3, 16].

The Ginzburg-Landau potential is parameterized 


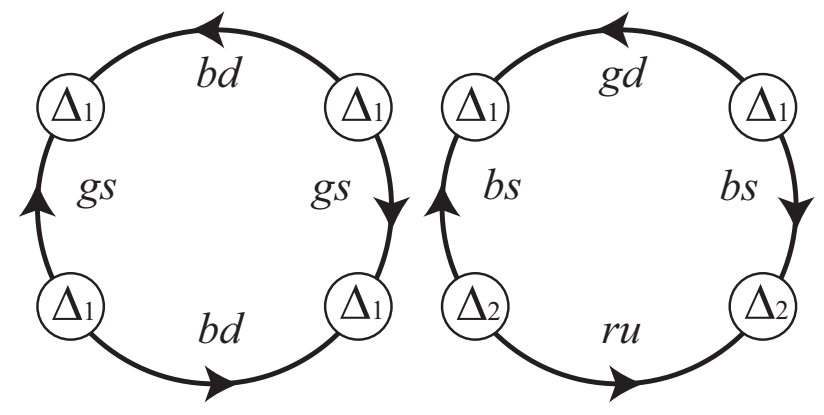

FIG. 20: Diagrams contributing to the $\Delta_{1}^{4}$ and $\Delta_{1}^{2} \Delta_{2}^{2}$ terms in the Ginzburg Landau potential (32).

as 16

$$
\begin{aligned}
\Omega= & \alpha\left(\Delta_{1}^{2}+\Delta_{2}^{2}+\Delta_{3}^{2}\right) \\
& +\epsilon\left(\Delta_{1}^{2}+\Delta_{2}^{2}\right)+\frac{1}{3} \eta\left(-2 \Delta_{1}^{2}+\Delta_{2}^{2}+\Delta_{3}^{2}\right) \\
& +\beta_{1}\left(\Delta_{1}^{2}+\Delta_{2}^{2}+\Delta_{3}^{2}\right)^{2}+\beta_{2}\left(\Delta_{1}^{4}+\Delta_{2}^{4}+\Delta_{3}^{4}\right),
\end{aligned}
$$

where $\alpha=\alpha_{0}\left(T-T_{\mathrm{c}}\right) / T_{\mathrm{c}}$ and where the coefficients $\epsilon$ and $\eta$ are proportional to $M_{s}^{2}$ and $\mu_{e}$ respectively and are therefore present only if $M_{s} \neq 0$. The form of the $\epsilon$ and $\eta$ terms was derived in Ref. 16] for QCD at asymptotic densities. In the Ginzburg-Landau limit, in which all gap parameters are small, color neutrality occurs with $\mu_{3}$ and $\mu_{8}$ vanishingly small [16], and electrical neutrality requires a nonzero $\mu_{e}$, of order $M_{s}^{2} / 4 \mu$ as in unpaired quark matter. At low temperatures, the most important consequences of $M_{s}$ and $\mu_{e}$ (and also $\mu_{3}$ and $\mu_{8}$ ) derive from the stress they put on pairing, as they (seek to) push Fermi momenta apart. Near $T_{c}, T \gg \Delta$. And, we are working at $M_{s}^{2} / \mu \ll T_{c}$, where the thermal smearing of the Fermi distributions is much greater than the splittings between Fermi momenta. In this regime, the effects of $M_{s}^{2}$ or $\mu_{e}$ on the pairing of two quarks do not arise from the splitting between their Fermi momenta, as this is negligible. The effects of $M_{s}^{2}$ or $\mu_{e}$ arise from the change in the average Fermi momenta of the two quarks, and hence in the density of states, that $M_{s}^{2}$ or $\mu_{e}$ induces. For example, $M_{s}^{2}$ depresses the average Fermi momenta of $u-s$ and $d-s$ pairs, but does not affect $u-d$ pairs. This explains the form of the $\epsilon$ term in (32). On the other hand, $\mu_{e}>0$ increases the average Fermi momenta of $d$ $s$ pairs, while decreasing that of $u-d$ and $u-s$ pairs. This explains the form of the $\eta$ term.

In Ref. [3] it is shown that, in the weak coupling regime, $\beta_{1}=\beta_{2}$. This result is valid beyond weak coupling, however, as all that is required to demonstrate it is the pairing ansatz (3) and the mean field approximation. The quarks in the $2 \times 2$ blocks contribute through diagrams like the first in Fig. 20, leading to a contribution proportional to $\Delta_{1}^{4}+\Delta_{2}^{4}+\Delta_{3}^{4}$. The quarks in the $3 \times 3$ block contribute through diagrams like both the first and second in Fig. 201 leading to a contribution proportional to $\Delta_{1}^{4}+\Delta_{2}^{4}+\Delta_{3}^{4}+2 \Delta_{1}^{2} \Delta_{2}^{2}+2 \Delta_{2}^{2} \Delta_{3}^{2}+2 \Delta_{1}^{2} \Delta_{3}^{2}$. Adding all the diagrams, we conclude that $\beta_{1}=\beta_{2}$.

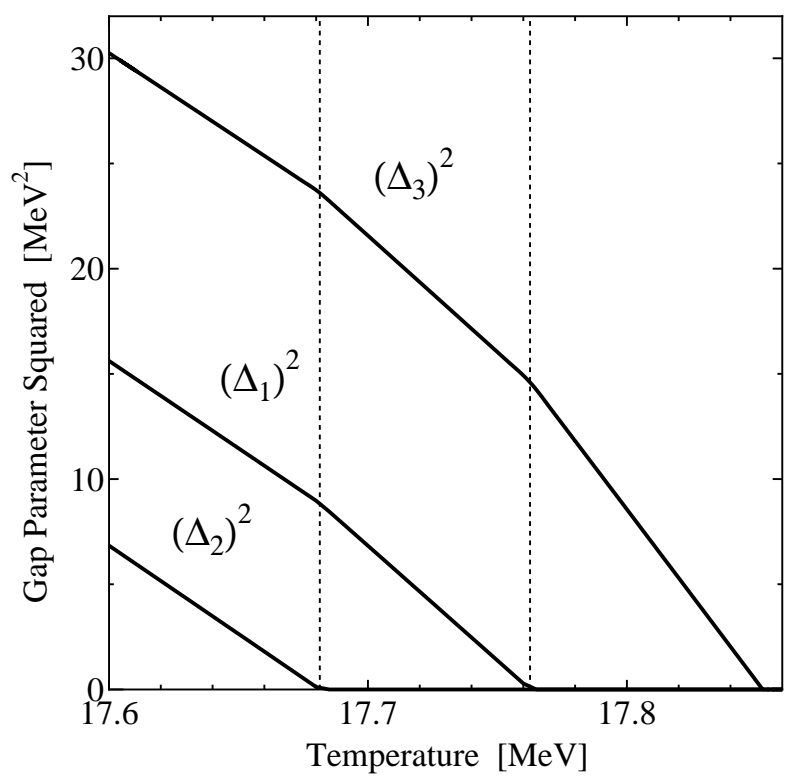

FIG. 21: Gap parameters squared versus $T$ at $M_{s}=30 \mathrm{MeV}$, namely $M_{s}^{2} / \mu=1.8 \mathrm{MeV}$. This figure should be compared to the "schematic illustration" given in Fig. 2 of Ref. 16]. We see the three phase transitions separating the CFL, dSC, 2SC and unpaired phases.

We now wish to confirm that (32) correctly describes the $M_{s}$-dependent physics in our model. We do so by extracting the ratio

$$
\zeta \equiv \frac{\epsilon}{\eta}
$$

from our results in three independent ways. If (32) is correct - that is if there are no $M_{s}$-dependent terms missed - the three extractions of $\zeta$ should agree. We see in the phase diagrams of Figs. 10 16] and 17 that the slopes of the three transition temperatures (i.e. $d T_{c} / d\left(M_{s}^{2} / \mu\right)$ at $\left.M_{s}^{2} / \mu=0\right)$ are different, with that for the $\Delta_{3} \rightarrow$ 0 critical temperature the shallowest and that for the $\Delta_{2} \rightarrow 0$ critical temperature the steepest. The ratios of these slopes can be extracted from the Ginzburg-Landau free energy $\Omega$ of (32) and are given by

$$
1:(6 \zeta-5):(6 \zeta+4)
$$

where we have used $\beta_{1}=\beta_{2}$. We can also read the slopes directly from our phase diagrams. The $\Delta_{0}=25 \mathrm{MeV}$ phase diagram of Fig. 1yields the ratios

$$
1: 10.1: 19.1
$$

which can be used to obtain two independent extractions of $\zeta$, one from $1:(6 \zeta-5)=1: 10.1$ and the other from $1:(6 \zeta+4)=1: 19.1$. The two are in perfect agreement, with both yielding $\zeta=2.52$ for $\Delta_{0}=25 \mathrm{MeV}$.

A third extraction can be obtained from Fig. 21] upon realizing that, according to (32), in the CFL phase where 
all three gap parameters are nonzero $\zeta$ is given by the ratio

$$
\frac{\Delta_{3}^{2}-\Delta_{2}^{2}}{\Delta_{1}^{2}-\Delta_{2}^{2}}
$$

This ratio can be extracted at $M_{s}^{2} / \mu=1.8 \mathrm{MeV}$ from Fig. 21. We have done this extraction at a number values of $M_{s}^{2} / \mu$ and fitted the results as

$$
\frac{\Delta_{3}^{2}-\Delta_{2}^{2}}{\Delta_{1}^{2}-\Delta_{2}^{2}}=2.52+36.2\left(\frac{M_{s}}{\mu}\right)^{2}+1.02 \times 10^{3}\left(\frac{M_{s}}{\mu}\right)^{4}
$$

which leads us to conclude for the third time that $\zeta=$ 2.52 for $\Delta_{0}=25 \mathrm{MeV}$. In (37) we have extracted the $M_{s}^{2} / \mu^{2}$ and $M_{s}^{4} / \mu^{4}$ corrections to the ratio (36), in addition to extracting $\zeta$. The coefficients we have obtained confirm that these higher order terms are negligible as long as $M_{s}^{2} / \mu^{2} \ll T_{c} / \mu$ or, equivalently given (11), $M_{s}^{2} / \mu \ll \Delta_{0}$. This indicates yet again that the GinzburgLandau free energy (32) provides a good approximation to our results in the regime where it should be valid.

Upon comparing our Fig. 21 with the schematic illustration given in Fig. 2 of Ref. 16], there can already be little doubt that the Ginzburg-Landau potential (32) correctly describes the results that we have obtained by solving the full gap and neutrality equations numerically, for $T \sim T_{c}$ and for $M_{s}^{2} / \mu^{2} \ll T_{c} / \mu$. The agreement between our three extractions of $\zeta$ makes this point quantitatively.

We can also use Fig. 21 to check that our numerical results are consistent with $\beta_{1}=\beta_{2}$. The Ginzburg-Landau potential (32) can first be used to show that in the CFL (and dSC) phases in Fig. 21] the slopes of the lines for the three (two) nonzero gap parameters are the same. Our results clearly satisfy this. Next, the Ginzburg-Landau potential (32) can be used to show that the ratio of the slopes of the gap parameters in the CFL phase in Fig. 21 to those in the dSC phase is $\left(2 \beta_{1}+\beta_{2}\right) /\left(3 \beta_{1}+\beta_{2}\right)$, and the ratio of those in the $\mathrm{dSC}$ phase to that in the $2 \mathrm{SC}$ phase is $\left(\beta_{1}+\beta_{2}\right) /\left(2 \beta_{1}+\beta_{2}\right)$. These ratios cannot be extracted very accurately from Fig. 21] given the narrow windows within which the dSC and 2SC phases are found. However, they are consistent with $3 / 4$ and $2 / 3$, corresponding to $\beta_{1}=\beta_{2}$.

In Ref. [16], the ratio $\zeta$ is calculated using weak coupling methods, valid at asymptotic densities. The weak coupling result is $\zeta=2$. From our numerical results, we have found $\zeta=2.52$ at $\Delta_{0}=25 \mathrm{MeV}$. We also find $\zeta=2.69$ at $\Delta_{0}=40 \mathrm{MeV}$ and $\zeta=3.87$ at $\Delta_{0}=100 \mathrm{MeV}$, extracting $\zeta$ from the ratio (36) as in (37). At weak coupling, $\zeta=2$ and the $\Delta_{3}^{2}$ and $\Delta_{2}^{2}$ lines are equidistant from the $\Delta_{1}^{2}$ line in the CFL phase region of Fig. 21. As the coupling gets stronger, the $\Delta_{1}^{2}$ and $\Delta_{2}^{2}$ lines move downward/leftward, further away from the $\Delta_{3}^{2}$ line, and the ratio $\zeta$ increases.

Now that we are convinced that the $\epsilon$ and $\eta$ terms fully describe the $M_{s}$-dependent physics in our model at small $M_{s}$, we calculate $\epsilon$ and $\eta$, from diagrams like those in Fig. 22 After some calculation, the result is

$$
\begin{aligned}
& \epsilon=\frac{M_{s}^{2}}{4 \pi^{2} \mu} \int_{0}^{\Lambda} d p p^{2}\left\{\frac{1}{(p-\mu)^{2}} \tanh \left(\frac{p-\mu}{2 T_{c}}\right)-\frac{1}{(p+\mu)^{2}} \tanh \left(\frac{p+\mu}{2 T_{c}}\right)\right. \\
& \left.-\frac{\mu}{2 T_{c} p(p-\mu)}\left[\cosh \left(\frac{p-\mu}{T_{c}}\right)\right]^{-2}-\frac{\mu}{2 T_{c} p(p+\mu)}\left[\cosh \left(\frac{p+\mu}{T_{c}}\right)\right]^{-2}\right\} \\
& \eta=\frac{\mu_{e}}{2 \pi^{2}} \int_{0}^{\Lambda} d p p^{2}\left\{\frac{1}{(p-\mu)^{2}} \tanh \left(\frac{p-\mu}{2 T_{c}}\right)-\frac{1}{(p+\mu)^{2}} \tanh \left(\frac{p+\mu}{2 T_{c}}\right)\right. \\
& \left.-\frac{1}{2 T_{c}(p-\mu)}\left[\cosh \left(\frac{p-\mu}{T_{c}}\right)\right]^{-2}+\frac{1}{2 T_{c}(p+\mu)}\left[\cosh \left(\frac{p+\mu}{T_{c}}\right)\right]^{-2}\right\} \text {. }
\end{aligned}
$$

In the weak-coupling limit, $T_{c} \ll \mu$ and the integrals are dominated by $p$ within $T_{c}$ of $\mu$. In this limit, it is easy to check that $\zeta=\epsilon / \eta=M_{s}^{2} / 2 \mu \mu_{e}$, which yields $\zeta=2$ since $\mu_{e}=M_{s}^{2} / 4 \mu$. At non-infinitesimal coupling, for example taking $\Delta_{0}=25 \mathrm{MeV}$ and reading the corresponding $T_{c}$ from Fig. 11 we can evaluate $\epsilon$ and $\eta$. We find $\zeta=2.55$ if we use $\mu_{e}=M_{s}^{2} / 4 \mu$, and if instead we obtain $\mu_{e}$ from our numerical results, we find $\zeta=2.52$. Taking $T_{c}$ and $\mu_{e}$ from our numerical results and evaluating $\zeta$ using (3839) we find $\zeta=2.52,2.65,3.84$ for $\Delta_{0}=25,40,100 \mathrm{MeV}$ respectively. From our numerical results, we have found $\zeta=2.52,2.69,3.87$ at these values of $\Delta_{0}$, extracting $\zeta$ from the ratio (36) as in (37). The agreement between these determinations is a confirmation of the accuracy of our numerical methods.

It is nice to see how quantitatively well the GinzburgLandau approximation describes the physics near $T_{c}$ in our model, as we have demonstrated. Furthermore, the 


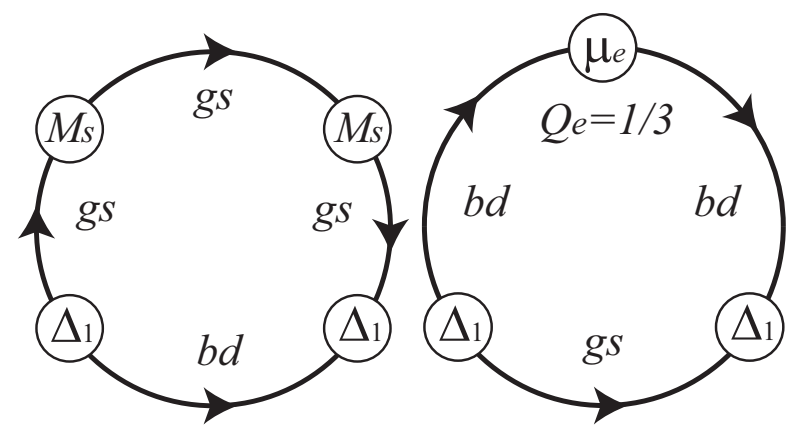

FIG. 22: Diagrams contributing to the $\epsilon$ and $\eta$ terms in the Ginzburg Landau potential (32).

value of one of the two ratios of coefficients that we have investigated, $\beta_{1} / \beta_{2}=1$, is the same in our model and in QCD at asymptotic densities. And, the value of the other ratio $\zeta=\epsilon / \eta$ is comparable in our model to its value in QCD at asymptotic densities for $\Delta_{0}=25$ and $40 \mathrm{MeV}$, becoming significantly larger only for quite strong coupling, as at $\Delta_{0}=100 \mathrm{MeV}$.

\section{IMPLICATIONS AND OPEN QUESTIONS}

The phase diagrams shown in Figs. 11 16] and 17 constitute the central results of this paper. They could be used in one of two different ways. If future theoretical advances constrain the $\mu$-dependent values of $\Delta_{0}$ and $M_{s}$ more tightly than at present, these phase diagrams (and those that interpolate between them for other values of $\Delta_{0}$ ) could be used to construct the phase diagram of nature. Or, if future astrophysical observations teach us that the phase diagram of nature must have certain features, for example must or must not include a certain phase, then the phase diagrams we have constructed could be used to draw inferences about the magnitudes of $\Delta_{0}$ and $M_{s}$.

In thinking about the future phenomenological use of the phase diagrams that we have found, their complexity raises concerns. However, in most astrophysical contexts compact stars have temperatures much less than $1 \mathrm{MeV}$. At such low temperatures, which can be thought of as $T=0$ in a QCD context, the phase diagrams are more manageable. We have the CFL phase at asymptotic densities, with the gCFL phase taking over at lower densities, when $\mu<M_{s}^{2} / 2 \Delta$. If $\Delta_{0}$ lies at the large end of its estimated range $10 \mathrm{MeV}<\Delta_{0}<100 \mathrm{MeV}$, it seems likely that hadronic matter will take over from gCFL (or even from CFL) meaning that the complexities that we have found in our phase diagrams at larger $M_{s}^{2} / \mu$ will likely be superseded by the transition to the hadronic phase.

If $\Delta_{0}$ lies at the lower end of its allowed range, then in Fig. 11 we find a straightforward transition to "unpaired" quark matter as the density is decreased further and $M_{s}^{2} / \mu$ increases beyond the gCFL window. If we extend our pairing ansatz, we will certainly find some pair- ing in this regime at sufficiently low temperature. For example, perhaps weak pairing between quarks with the same flavor plays a role [7, 41], or perhaps it is the crystalline color superconducting phase [37, 42] that takes over from gapless CFL at lower densities. Recent developments 42] make the crystalline color superconducting phase look like the most viable contender 6 ].

The one astrophysical context in which the full complexity of the phase diagrams that we have analyzed must be faced head-on is the physics of the proto-neutron star formed during a supernova, and in particular of the propagation of neutrinos therein. Phenomena encoded in the phase diagrams that we have analyzed could ultimately result in observable consequences in the time-of-arrival distribution of the neutrinos detected from a future supernova [27, 29, 43]. The phenomenological implications of the complexity of the phase diagrams (with many phase transition lines, the doubly critical point, the tricritical point and the insulator to metal transition as the CFL phase is heated) will have to be thought through in this context. The analytic treatment of the insulator to metal crossover and the Ginzburg-Landau analysis of the physics near $T_{c}$ for small $M_{s}^{2} / \mu T_{c}$ that we have presented in Sections $\nabla$ and $\nabla I$ could prove valuable in this context.

Our analysis leaves many open avenues of investigation that must still be followed to their conclusions. The effects if gauge field fluctuations must be investigated, as must those of $K^{0}$-condensation in the gapless CFL phase. The effects of the 't Hooft interaction should be included, and the quark masses should be treated as dynamical condensates to be solved for, rather than as parameters. The pairing ansatz should be generalized, for example to allow for a comparison between the free energies of the gapless CFL and crystalline phases in three-flavor QCD.

Finally, the stability of the gapless CFL phase needs to be investigated further, along the lines of Ref. [44]. These authors find that the g2SC phase is unstable, as is the $2 \mathrm{SC}$ phase near the $2 \mathrm{SC} \rightarrow \mathrm{g} 2 \mathrm{SC}$ transition. This instability could reflect the known instability of the g2SC phase with respect to a mixed phase of charged components 45], it could reflect an instability with respect to the crystalline color superconducting phase, or it could reflect the existence of some inhomogeneous phase yet to be discovered. We know that the gCFL phase is stable with respect to mixed phases, except perhaps at the largest values of $M_{s}^{2} / \mu$ where it is found in our phase diagrams 6, 46]. It seems likely that at these large values of $M_{s}^{2} / \mu$ the gCFL phase may anyway be superseded by the crystalline phase $[6,42$. This could be clarified either by a direct free energy comparison of these two phases, or by a stability analysis of the gCFL phase as in Ref. [4].

\section{Acknowledgments}

We acknowledge helpful conversations with M. Alford, J. Bowers, M. Forbes, T. Hatsuda, M. Huang, J. Kundu, 
T. Schaefer, I. Shovkovy and M. Tachibana. C. K. and K. R. thank the Institute for Nuclear Theory in Seattle for its hospitality. K. F. is grateful to D. Rischke for hospitality at the University of Frankfurt during the completion of this work. K. F. was supported by Japan Society for the Promotion of Science for Young Scientists. This research was supported in part by the U.S. Department of Energy (D.O.E.) under cooperative research agreement \#DF-FC02-94ER40818.
[1] For reviews, see K. Rajagopal and F. Wilczek, arXiv:hep-ph/0011333 M. G. Alford, Ann. Rev. Nucl. Part. Sci. 51, 131 (2001) arXiv:hep-ph/0102047; G. Nardulli, Riv. Nuovo Cim. 25N3, 1 (2002) arXiv:hep-ph/0202037; S. Reddy, Acta Phys. Polon. B 33, 4101 (2002) arXiv:nucl-th/0211045; T. Schäfer, arXiv:hep-ph/0304281 M. Alford, arXiv:nucl-th/0312007

[2] M. G. Alford, K. Rajagopal and F. Wilczek, Nucl. Phys. B 537, 443 (1999) arXiv:hep-ph/9804403.

[3] K. Iida and G. Baym, Phys. Rev. D 63, 074018 (2001) [Erratum-ibid. D 66, $059903 \quad$ (2002)] arXiv:hep-ph/0011229;

[4] M. Alford and K. Rajagopal, JHEP 0206, 031 (2002) arXiv:hep-ph/0204001.

[5] M. Alford, C. Kouvaris and K. Rajagopal, Phys. Rev. Lett. 92, 222001 (2004) arXiv:hep-ph/0311286.

[6] M. Alford, C. Kouvaris and K. Rajagopal, Phys. Rev. D., to appear, arXiv:hep-ph/0406137

[7] A. Schmitt, Q. Wang and D. H. Rischke, Phys. Rev. D 66, 114010 (2002) arXiv:nucl-th/0209050.

[8] B. I. Halperin, T. C. Lubensky and S. k. Ma, Phys. Rev. Lett. 32, 292 (1974).

[9] T. Matsuura, K. Iida, T. Hatsuda and G. Baym, Phys. Rev. D 69, 074012 (2004) arXiv:hep-ph/0312042.

[10] I. Giannakis, D. f. Hou, H. c. Ren and D. H. Rischke, arXiv:hep-ph/0406031

[11] A. W. Steiner, S. Reddy and M. Prakash, Phys. Rev. D 66, 094007 (2002) arXiv:hep-ph/0205201.

[12] P. F. Bedaque and T. Schafer, Nucl. Phys. A 697, 802 (2002) arXiv:hep-ph/0105150; D. B. Kaplan and S. Reddy, Phys. Rev. D 65, 054042 (2002) arXiv:hep-ph/0107265; A. Kryjevski, D. B. Kaplan and T. Schafer, arXiv:hep-ph/0404290

[13] A. Kryjevski and T. Schaefer, arXiv:hep-ph/0407329 A. Kryjevski and D. Yamada, arXiv:hep-ph/0407350

[14] W. V. Liu, F. Wilczek and P. Zoller, arXiv:cond-mat/0404478

[15] S. B. Rüster, I. A. Shovkovy and D. H. Rischke, arXiv:hep-ph/0405170

[16] K. Iida, T. Matsuura, M. Tachibana and T. Hatsuda, arXiv:hep-ph/0312363

[17] K. Iida and G. Baym, Phys. Rev. D 66, 014015 (2002) arXiv:hep-ph/0204124.

[18] M. G. Alford, J. Berges and K. Rajagopal, Nucl. Phys. B 558, 219 (1999) arXiv:hep-ph/9903502.

[19] M. G. Alford, J. Berges and K. Rajagopal, Nucl. Phys. B 571, 269 (2000) arXiv:hep-ph/9910254.

[20] D. K. Hong, Nucl. Phys. B 582, 451 (2000) arXiv:hep-ph/9905523; N. J. Evans, J. Hormuzdiar, S. D. H. Hsu and M. Schwetz, Nucl. Phys. B 581, 391 (2000) arXiv:hep-ph/9910313.

[21] R. Rapp, T. Schafer, E. V. Shuryak and M. Velkovsky, Annals Phys. 280, 35 (2000) arXiv:hep-ph/9904353.
[22] T. Schafer, Nucl. Phys. B 575, 269 (2000) arXiv:hep-ph/9909574.

[23] C. Manuel and M. H. G. Tytgat, Phys. Lett. B 479, 190 (2000) arXiv:hep-ph/0001095.

[24] T. Schafer, Phys. Rev. D 65, 094033 (2002) arXiv:hep-ph/0201189.

[25] D. T. Son and M. A. Stephanov, Phys. Rev. D 61, 074012 (2000) arXiv:hep-ph/9910491; Phys. Rev. D 62, 059902 (2000) arXiv:hep-ph/0004095.

[26] D. K. Hong, T. Lee and D. P. Min, Phys. Lett. B 477, 137 (2000) arXiv:hep-ph/9912531; S. R. Beane, P. F. Bedaque and M. J. Savage, Phys. Lett. B 483, 131 (2000) arXiv:hep-ph/0002209; T. Schafer, Phys. Rev. D 65, 074006 (2002) arXiv:hep-ph/0109052.

[27] S. Reddy, M. Sadzikowski and M. Tachibana, Nucl. Phys. A 714, 337 (2003) arXiv:nucl-th/0203011.

[28] P. Jaikumar, M. Prakash and T. Schafer, Phys. Rev. D 66, 063003 (2002) arXiv:astro-ph/0203088; I. A. Shovkovy and P. J. Ellis, Phys. Rev. C 66, 015802 (2002) arXiv:hep-ph/0204132.

[29] J. Kundu and S. Reddy, arXiv:nucl-th/0405055

[30] P. Amore, M. C. Birse, J. A. McGovern and N. R. Walet, Phys. Rev. D 65, 074005 (2002) arXiv:hep-ph/0110267.

[31] A. Gerhold and A. Rebhan, Phys. Rev. D 68, 011502 (2003) arXiv:hep-ph/0305108; A. Kryjevski, Phys. Rev. D 68, 074008 (2003) arXiv:hep-ph/0305173; D. D. Dietrich and D. H. Rischke, Prog. Part. Nucl. Phys. 53, 305 (2004) arXiv:nucl-th/0312044.

[32] K. Rajagopal and F. Wilczek, Phys. Rev. Lett. 86, 3492 (2001) arXiv:hep-ph/0012039.

[33] J. M. Cornwall, R. Jackiw and E. Tomboulis, Phys. Rev. D 10, 2428 (1974).

[34] M. Buballa and M. Oertel, Nucl. Phys. A 703, 770 (2002) arXiv:hep-ph/0109095; F. Neumann, M. Buballa and M. Oertel, Nucl. Phys. A 714, 481 (2003) arXiv:hep-ph/0210078.

[35] I. Shovkovy and M. Huang, Phys. Lett. B 564, 205 (2003) arXiv:hep-ph/0302142; M. Huang and I. Shovkovy, arXiv:hep-ph/0307273

[36] E. Gubankova, W. V. Liu and F. Wilczek, Phys. Rev. Lett. 91, 032001 (2003).

[37] P. Fulde and R. A. Ferrell, Phys. Rev. 135, A550 (1964); S. Takada and T. Izuyama, Prog. Theor. Phys. 41, 635 (1969); M. G. Alford, J. A. Bowers and K. Rajagopal, Phys. Rev. D 63, 074016 (2001) arXiv:hep-ph/0008208; J. .A. Bowers, J. Kundu, K. Rajagopal and E. Shuster, Phys. Ref. D 64, 014024 (2001) arXiv:hep-ph/0101067; J. Kundu and K. Rajagopal, Phys. Rev. D 65, 094022 (2002) arXiv:hep-ph/0112206; J. A. Bowers and K. Rajagopal, Phys. Rev. D 66, 065002 (2002) arXiv:hep-ph/0204079; R. Casalbuoni and G. Nardulli, Rev. Mod. Phys. 263, 320 (2004) arXiv:hep-ph/0305069.

[38] G. Sarma, J. Phys. Chem. Solids 24, 1029 (1963). 
[39] M. G. Alford, J. Berges and K. Rajagopal, Phys. Rev. Lett. 84, 598 (2000) arXiv:hep-ph/9908235.

[40] A. Sedrakian and U. Lombardo, Phys. Rev. Lett. 84, 602 (2000) arXiv:nucl-th/9907076.

[41] M. Iwasaki and T. Iwado, Phys. Lett. B 350, 163 (1995); M. G. Alford, K. Rajagopal and F. Wilczek, Phys. Lett. B 422, 247 (1998) arXiv:hep-ph/9711395; T. Schafer, Phys. Rev. D 62, 094007 (2000) arXiv:hep-ph/0006034; M. Buballa, J. Hosek and M. Oertel, Phys. Rev. Lett. 90, 182002 (2003) arXiv:hep-ph/0204275; M. G. Alford, J. A. Bowers, J. M. Cheyne and G. A. Cowan, Phys. Rev. D 67, 054018 (2003) arXiv:hep-ph/0210106; A. Schmitt, arXiv:nucl-th/0405076

[42] R. Casalbuoni, M. Ciminale, M. Mannarelli, G. Nardulli, M. Ruggieri and R. Gatto, arXiv:hep-ph/0404090
[43] G. W. Carter and S. Reddy, Phys. Rev. D 62, 103002 (2000) arXiv:hep-ph/0005228.

[44] M. Huang and I. A. Shovkovy, arXiv:hep-ph/0407049

[45] P. F. Bedaque, H. Caldas and G. Rupak, Phys. Rev. Lett. 91, 247002 (2003) arXiv:cond-mat/0306694; M. M. Forbes, E. Gubankova, W. V. Liu and F. Wilczek, arXiv:hep-ph/0405059 S. Reddy and G. Rupak, arXiv:nucl-th/0405054

[46] M. Alford, C. Kouvaris and K. Rajagopal, arXiv:hep-ph/0407257

[47] We have learned from them in private communications that the authors of Ref. 15] now find an ordering of phase transitions in agreement with our results and the Ginzburg-Landau analysis. 